\title{
MAXWELL FISHEYE LENS AS A WAVEGUIDE CROSSING FOR INTEGRATED PHOTONICS
}

\author{
By \\ Joy Carleen Garnett
}

Thesis

Submitted to the Faculty of the

Graduate School of Vanderbilt University

in partial fulfillment of the requirements

for the degree of

\section{MASTER OF SCIENCE}

in

Interdisciplinary Materials Science

August, 2013

Nashville, Tennessee

Approved:

Dr. Norman Tolk

Dr. Jason Gage Valentine 


\section{ACKNOWLEDGMENTS}

This research project would not have been possible without the support of many people. The author wishes to express her gratitude to Professors Dr. Jason Valentine and Dr. Norman Tolk who have been abundantly helpful and offered invaluable assistance, support and guidance. Deepest gratitude are also due to the members of VINSE staff, Dr. Anthony Hmelo, Dr. Ben Schmidt, Dr. Bo Choi, and Dr. Dmitry Koktysh without whose knowledge and assistance this study would not have been successful.

The author would also like to acknowledge the contribution of Hiram Conley for his hours of troubleshooting and support. Special thanks also to all her graduate friends, especially group members; Stephanie Gilbert-Corder, Jennifer Ann Jones, Dr. Halina Krzyzanowska, Zeynab Jarrahi, Parikshit Moitra, and Jason Greaving for sharing the literature and invaluable assistance, not forgetting her best friends who always been there.

The author would also like to convey thanks to NASA under contract NNX09AW06A, NSF, GEM and Vanderbilt University for providing the financial means and laboratory facilities.

The author wishes to express her love and gratitude to her significant other, Michael Hehnen and her beloved families for their understanding endless love through the duration of her studies. 
TABLE OF CONTENTS

Page

ACKNOWLEDGMENTS $\ldots \ldots \ldots \ldots \ldots \ldots \ldots$ ii

LIST OF TABLES $\ldots \ldots \ldots \ldots \ldots \ldots \ldots \ldots \ldots \ldots \ldots$ iv

LIST OF FIGURES $\ldots \ldots \ldots \ldots \ldots \ldots \ldots \ldots \ldots \ldots \ldots$

I Introduction $\ldots \ldots \ldots \ldots \ldots \ldots \ldots \ldots \ldots \ldots$

I.1 Fundamental Limits to Integrated Circuit Growth . . . . . . . . . . . 1

I.2 Motivation for Integrated Silicon Photonics . . . . . . . . . . . . 2

I.3 Review of Waveguide Crossings . . . . . . . . . . . . . . . . . 4

II Proposed Maxwell Fisheye Waveguide Crossing Design $\ldots \ldots \ldots \ldots$

II.1 Background of Maxwell Fisheye Lens _ . . . . . . . . . . . . . . . 14

II.2 Derivation of Maxwell Fisheye Lens Refractive Index Profile . . . . . . 15

II.2.1 Ray Optics Approach . . . . . . . . . . . . . . . . . . . 15

II.2.2 Transformation Optics Approach . . . . . . . . . . . . . 16

III Modeling and Performance $\ldots \ldots \ldots \ldots \ldots \ldots$

III.1 2D Simulation of MFE Crossing Performance . . . . . . . . . . 20

III.2 3D Simulation of MFE Crossing Performance $\ldots \ldots \ldots \ldots \ldots$

IV Fabrication Protocols $\ldots \ldots \ldots \ldots \ldots \ldots \ldots \ldots$

IV.1 Grayscale Bitmap to Fabricated Device Height Translation . . . . . . . . 28

IV.2 Focused Ion Beam Grayscale Lithography Procedure . . . . . . . . . . . 29

IV.3 Grayscale Electron-Beam Lithography with RIE Pattern Transfer Procedure 31

V Conclusions and Future Work $\ldots \ldots \ldots \ldots \ldots \ldots$

A Appendix A: Slab Waveguide Theory $\ldots \ldots \ldots \ldots \ldots \ldots$

A.1 Symmetric Waveguides . . . . . . . . . . . . . . 37

A.2 Asymmetric Waveguides . . . . . . . . . . . . . . . 41

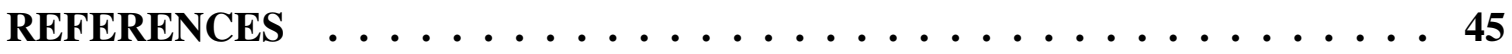




\section{LIST OF TABLES}

Table

Page

I.1 Comparison of Material Properties for Use in On-chip Photonics . . . . 3 


\section{LIST OF FIGURES}

Figure

I.1 Schematic of PDWG Crossing $25 \ldots \ldots \ldots$

I.2 Effectiveness of PDWG Crossing Compared to Plain Waveguide Cross-

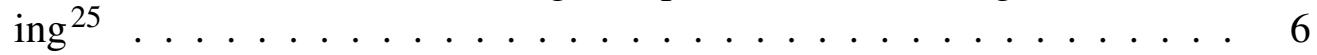

I.3 The Si/Polymer Waveguide Crossing Designed by Tsarev Viewed From the Bottom. Buried oxide and Si substrate are not shown. ${ }^{26} \ldots . . .7$

I.4 Various Crossings with Simulated Transmission Efficiencies ${ }^{27} \ldots \ldots$

I.5 Schematic of Proposed Bogaert's Waveguide Crossing $27 \ldots \ldots$

I.6 90 Degree Waveguide Crossings With and Without (Inset) Metamaterial Design. $^{28 \ldots \ldots \ldots \ldots \ldots \ldots} 11$

I.7 60 Degree Waveguide Crossings With and Without (Inset) Metamaterial Design. $^{28 \ldots \ldots \ldots \ldots \ldots \ldots} 12$

I.8 Simulated Transmission Efficiencies and Crosstalk Loss for Various Negative Index Metamaterial Crossing Angles. ${ }^{28} \ldots$. . . . . . . . . 12

II.1 An Example of the Maxwell Fisheye Lens Refractive Index Profile . . . 15

II.2 Fermat's Principle of Least Action in heterogenous refractive index ma-

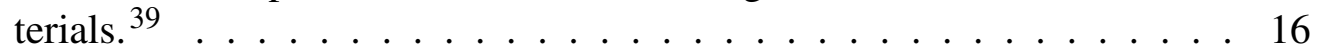

II.3 Optical transformation of the physical space Maxwell fisheye lens imag-

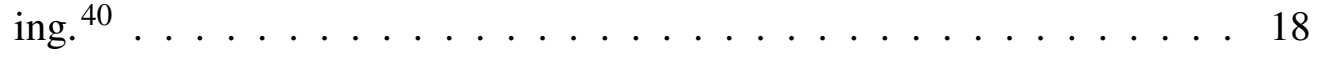

III.1 Electric Field Inside of a $6 \mu \mathrm{m}$ Wide Maxwell Fish Eye Crossing with One Auxillary Waveguide 34 . . . . . . . . . . . . . . 22

III.2 Electric Field Inside of a $6 \mu \mathrm{m}$ Wide Maxwell Fish Eye Crossing with Eight Auxillary Waveguides . . . . . . . . . . . . . . 22

III.3 Transmission Loss Plot of Maxwell Fish Eye Crossing with Up to Seven Equidistant Waveguides ... . . . . . . . . . . 23 
III.4 Modal Refractive Index Profile of a $10 \mu \mathrm{m}$ Radius Maxwell Fish Eye . . 24

III.5 3D Maxwell Fish Eye Modal Refractive Index With Respect to Tapered Slab Waveguide Height . . . . . . . . . . . . . . . . 25

III.6 Waveguide Height (in nm) for a $10 \mu \mathrm{m}$ Radius Maxwell Fish Eye . . . . 26

III.7 3D Maxwell Fish Eye Height Profile Realized By TE Tapered Slab Waveguides ...................... 27

IV.1 Grayscale Lithography with $D_{o}$ as the Largest Feature Height and $D_{i}$ as the Spatially Varying Feature Height ${ }^{41}$. . . . . . . . . . . . . . . . 29

IV.2 Example of Grayscale FIB Fabrication Resolution Capabilities in VINSE 30

IV.3 Grayscale FIB Fabrication of Maxwell Fisheye Waveguide Crossing ${ }^{34}$. 31

IV.4 Grayscale EBL-RIE Protocol on an SOI Substrate . . . . . . . . . . 33

IV.5 Comparison of Designed and FIB Fabricated Maxwell Fisheye Crossing Height Profile . . . . . . . . . . . . . . . . . . . 34

IV.6 Grayscale EBL-RIE of Arbitrary Radially Symmetric Design . . . . . . 35

A.1 Symmetric Waveguide Schematic ${ }^{46} \ldots \ldots . \ldots . \ldots$ 


\section{CHAPTER I}

\section{Introduction}

Silicon photonics is a relevant technology to solve several limitations of the integrated circuit industry. In this chapter, we review the limits of current integrated circuits and traditional interconnects. We will discuss the associated energy costs, the effect of parasitic resistance on performance, and the implication of the practical size limit on integrated circuitry. Next, we will discuss how silicon-based integrated optics solves these issues. In order to implement silicon-based integrated optics architectures, efficient waveguide crossings are required for high-density, high-performance hardware. Finally, we review the performance of recent waveguide crossings in the literature.

\section{I.1 Fundamental Limits to Integrated Circuit Growth}

In integrated circuitry, decreasing the feature size of on-chip devices improves cost, performance, and power. Over the years, transistor size has scaled down from $10 \mu \mathrm{m}$ with $\mathrm{Si}$ bipolar manufacturing to $30 \mathrm{~nm}$ achieved with complementary metal oxide semiconductor (CMOS) manufacturing technology. This trend of scaling transistor feature size has been

deemed Moore's Law ${ }^{1}$, which states that IC device density and performance doubles every year or every two years. To accomodate this increase in density, lithographic manufacturing tool costs escalated from $\$ 10,000$ to $\$ 35$ million, contributing to the $\$ 300$ billion industry worldwide $^{2}$. This has increased the cost per transistor several orders of magnitude ${ }^{3,4}$.

In addition to cost, another barrier for decreasing device feature dimension is the minimum practical size limit ${ }^{2,5}$. With transistor gates smaller than $20 \mathrm{~nm}$, current leaks from the source happen even when the transistor is in the off stage. A gate this size decreases the barrier height between the drain and the source. This is known as short channel effect (SEC) or drain-induced barrier lowereing. This phenomenon is about $1 / 5$ to $1 / 3$ of the total power budget in that it requires more energy for cooling and more cooling cir- 
cuitry, which increases cost.

Parasitic resistance and capacitance between devices on a high-density integrated circuit platform is also an issue even if the transistor size is above the minimum practical size limit ${ }^{6,7,8}$. As the distance between on-chip electronic devices decreases, the higher contact resistance and contact-to-gate capacitance is on the order of the on-chip channel and intrinsic resistance and capacitance ${ }^{9}$. To combat these limits for integrated circuit industry, the computing industry has turned to integrated optics architectures. Integrated optics eliminates the inefficiencies of electrical resistance and capacitance found in electronic-based integrated circuits by using photons instead of electricity. One of the major architectures in integrated optics is silicon photonics, which will be discussed in the next section.

\section{I.2 Motivation for Integrated Silicon Photonics}

Silicon photonics is a relevant technology to solve the limitations of the integrated circuit industry for several reasons ${ }^{10,11,12,13,14}$. First, silicon photonic device manufacturing is compatible with current well-characterized IC manufacturing technologies. This economy of scale enables mass-market low-cost photonics and ease of implementation into current circuitry. Second, high-quality silicon-on-insulator (SOI) wafers are readily available for waveguide circuitry. These wafers enable ideal optical confinement due to the difference in the refractive index of silicon $(n=3.5)$ and silicon dioxide $(n=1.5)$. With this large difference, the waveguide mode can be decreased to $0.1 \mu \mathrm{m}^{2}$ into waveguide devices the size of hundreds of nanometers, which are completely feasible with current IC manufacturing methods. These device sizes make efficient use of on-chip real estate. Third, silicon's properties are better suited for photonics applications compared to other materials, such as gallium arsenide, gallium nitride and germanium. The bandgaps and refractive indicies of four major semiconductors are shown in the table below. 


\begin{tabular}{ccccc}
\hline Material Properties & Silicon & Germanium & Gallium Nitride & Gallium Arsenide \\
\hline Bandgap (eV) at 300 K & $1.11 \mathrm{eV}$ & $0.66 \mathrm{eV}$ & $3.40 \mathrm{eV}$ & $1.43 \mathrm{eV}$ \\
\hline Bandgap (nm) at 300 K & $1117.0 \mathrm{~nm}$ & $1878.5 \mathrm{~nm}$ & $364.6 \mathrm{~nm}$ & $867.0 \mathrm{~nm}$ \\
\hline Refractive Index & $3.497 @ 1.2 \mu \mathrm{m}$ & $4.104 @ 2 \mu \mathrm{m}$ & $2.327 @ 1.2 \mu \mathrm{m}$ & $3.419 @ 1.4 \mu \mathrm{m}$ \\
\hline
\end{tabular}

Table I.1: Comparison of Material Properties for Use in On-chip Photonics

Due to low-cost manufacturing and favorable optical properties with available SOI substrates, a potential solution is to use optical interconnects in conjunction with electrical CMOS architectures ${ }^{15,16,17}$. Current interconnects made of copper can handle high on-chip data rates on the magnitude of $\mathrm{Gb} / \mathrm{s}$. However, these interconnects come at a high cost with as data rates increase. At higher data rates, these small microscale copper-based interconnects generate high heat, causing degradation. With higher heat, the cooling systems then require more power limiting available for the computation. This increased heat also limits the computational speed between on-chip processors. This is referred to as the communication bottleneck. To mitigate this communication bottleneck and divert power from cooling to computation, less heat needs to be generated. In order to do that, one must not only shift from using copper as an interconnect material but also shift from electronic interconnects to optical interconnects. One example architecture using optical interconnects is on an SOI platform ${ }^{18,19,20}$. With SOI optical interconnects, less heat is generated at higher data rates, eliminating the power drain needed for cooling and the subsequent communication bottleneck.

Low losses are important for all optical circuitry components with high refractive index differences. There are three major losses in waveguide devices: propagation, crosstalk, and insertion losses ${ }^{21,22}$. Typical propagation losses for silicon waveguides are between $0.1 \mathrm{~dB} / \mathrm{cm}$ and $3 \mathrm{~dB} / \mathrm{cm}$. In a simple waveguide crossing, these wide-angle components normally diffract from the intended waveguide to the unintended waveguide and the environment, creating crosstalk and environmental loss respectively. The way to minimize all 
of these losses is to reduce scattering from the devices by reducing waveguide sidewall and surface roughness through oxidation. This reduction depends on oxidation time, temperature, and environment. For example, in our fabrication protocol, annealing at $700^{\circ} \mathrm{C}$ for 2 hours in air smoothed the surface of a silicon waveguide without significant alteration from the original profile.

\section{I.3 Review of Waveguide Crossings}

An ideal waveguide crossing exhibits high transmission (97\%), low crosstalk (1\%), small footprint $\left(<100 \mu \mathrm{m}^{2}\right)$, and the ability to be integrated into CMOS fabrication techniques $^{23,24}$. In this section, we will discuss four separate optical waveguide crossing designs: a periodic dielectric waveguide crossing ${ }^{25}$, a silicon wire waveguide crossing utilizing adiabatic coupling ${ }^{26}$, a crossing utilizing parabolic wave expanders ${ }^{27}$, and an impedance matched negative refractive index material waveguide crossing ${ }^{28}$. For each waveguide crossing, we will review the related advantages and disadvantages. In the next section, we will highlight the advantages of our waveguide crossing over those in this section.

In 2010, a periodic dielectric waveguide (PDWG) crossing demonstrated little crosstalk into unintended waveguides and high transmission capability ${ }^{25}$. Unlike other crossings, this fabrication is compatible with simultaneous construction with other waveguide devices unlike other crossings. A schematic of this unique design is shown in Figure I.1. The authors designed a periodic dielectric waveguide crossing using an array of two materials with drastically different refractive indices is needed, which is evident in Figure I.1. The orange represents silicon $(n=3.5)$ and the light blue background represents the air $(n=1)$ surroundings. With these materials, he used a $90^{\circ}$ dieletric air cylinder array crossing with cylinders of radius $\mathrm{r}$ spaced a nm apart. In this case, $a$ is the spacing between the centers of the dielectric cylinders and $r$ is the radius of the dielectric cylinders, shown as orange circles in Figure I.1. In these periodic waveguides, light travels between two neighboring high- 
index cylinders. The waveguide crossing is air (low-index) to ensure low coupling with unintentional waveguides. This type of waveguide exhibits a broad bandwidth of several hundred nanometers while maintaining single mode propogation. To further facilitate transition from a $0.5 \mu \mathrm{m}$ wide ridge waveguide with traditional taper into the PDWG cylinder array, the authors rounded the incoming taper to alter the modal spot size to prevent coupling losses into the crossing system. This rounded incoming taper is shown in the inset of Figure I.1 and was shown to decrease coupling losses between the waveguide and the crossing. To evaluate performance of this PDWG crossing, Fan excited the bottom waveguide and compared its power $\left(P_{i n}\right)$ to the power of the top intentional output waveguide $\left(P_{T}\right)$ and the power of the left and right unintentional output waveguides $\left(P_{C}\right)$. These powers were compared to the input power to evaluate transmission and crosstalk experimentally.

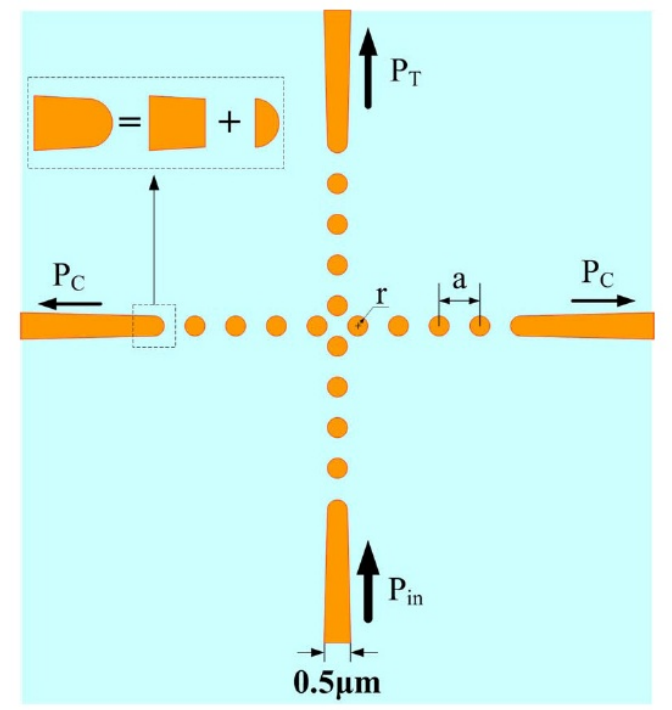

Figure I.1: Schematic of PDWG Crossing 25

The PDWG crossing produced high transmission and low crosstalk losses experimentally as shown on the left in Figure I.2. Between $1520 \mathrm{~nm}$ and $1600 \mathrm{~nm}$, the transmission remained between -1.1 and $-0.5 \mathrm{~dB}$, which equates to $77.6 \%$ and $89.1 \%$, respectively. In this same wavelength range, the crosstalk remained around $-40 \mathrm{~dB}$ or $0.004 \%$. The crosstalk and transmission is an improvement over the solid waveguide crossings results shown on the right in Figure I.2. Between the wavelengths of $1520 \mathrm{~nm}$ and $1600 \mathrm{~nm}$ wave- 
length, the transmission was around $-1.5 \mathrm{~dB}$ or $70.8 \%$. In this same wavelength range, the crosstalk of the plain waveguide crossing was much higher at $-16 \mathrm{~dB}$ or $2.5 \%$.
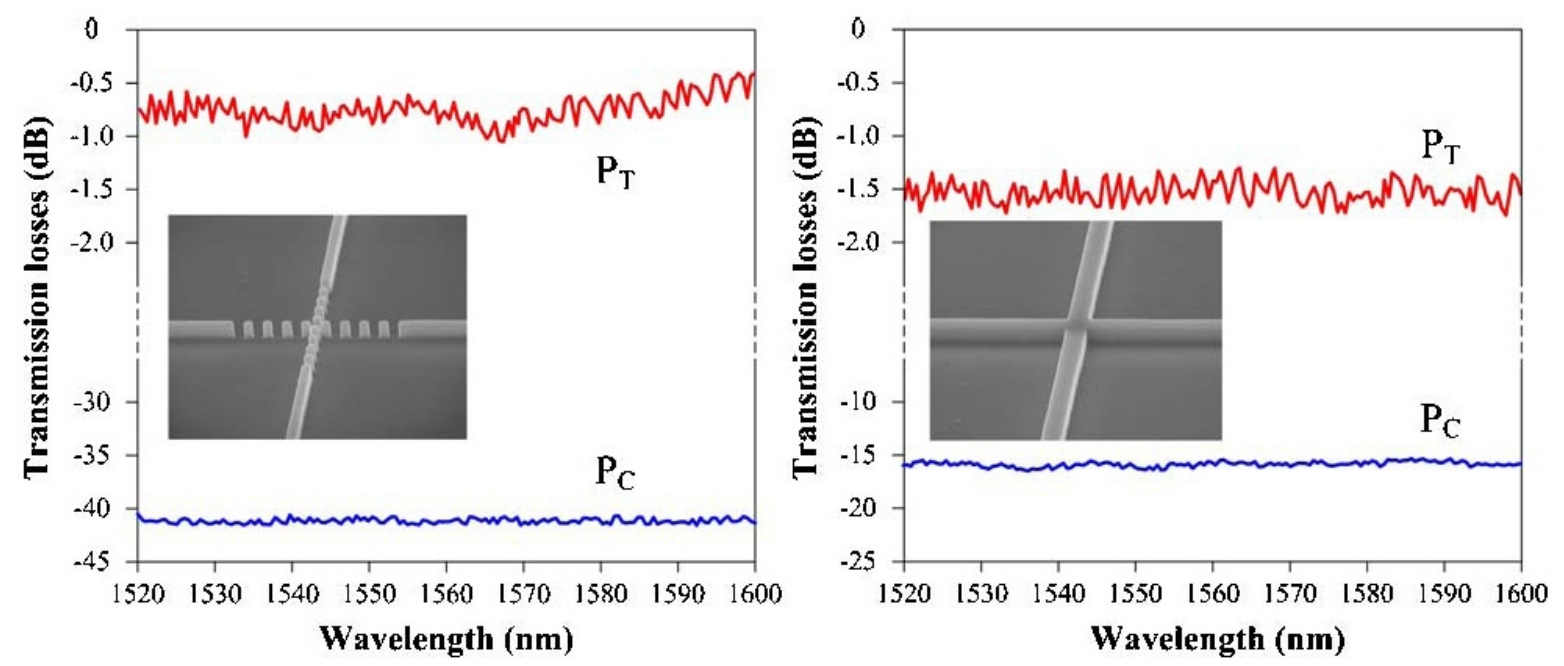

Figure I.2: Effectiveness of PDWG Crossing Compared to Plain Waveguide Crossing 25

While the performance of the PDWG crossing is high, there are several disadvantages to this approach. One disadvantage is the size requirement for such a crossing. The crossing itself is $16 \mu \mathrm{m}^{2}$ which is quite small, but the taper length required to ensure low loss has to be at least $10 \mu \mathrm{m}$. This increases the effective crossing area to $100 \mu \mathrm{m}^{2}$, which is too large to be efficient for high-density photonic networks. This size disadvantage becomes compounded when crossing several waveguides serially. In order to cross 6 waveguides with PDWG, at least $576 \mu \mathrm{m}^{2}$ is needed. This is too much area for a crossing scheme in high-density photonic networks. Another disadvantage is the performance depenence on polarization. While transverse electric (TE) and transverse magnetic (TM) fundamental modes would propagate through same periodic dielectric array, they have different band structures with a given PDWG structure. This difference in band structure would mean that the crossing would be effective over a different frequency range.

Another design exhibiting little crosstalk and loss to the environment is the silicon wire waveguide crossing proposed and simulated by Tsarev ${ }^{26}$. This waveguide crossing worked by vertical adiabatic interlayer coupling between a polymer waveguide and a ta- 
pered silicon waveguide with a silica barrier. Through his 3D optimization simulations, the authors were able to demostrate $98 \%$ transmission efficiency and a crosstalk of $-77 \mathrm{~dB}$ on an SOI substrate. They optimized this crossing by varying the silicon taper length and width, the distance between the silicon waveguides and crossed polymer tapers, and the upper polymer waveguide height and width.

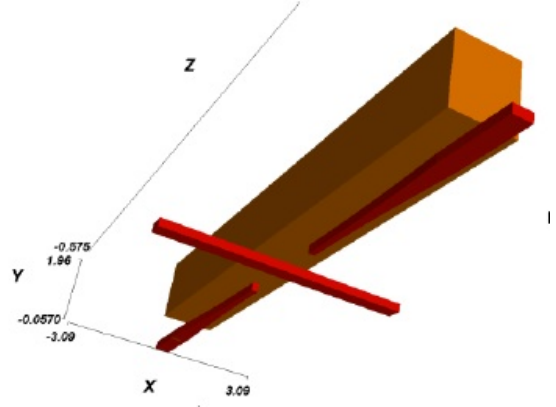

Figure I.3: The Si/Polymer Waveguide Crossing Designed by Tsarev Viewed From the Bottom. Buried oxide and Si substrate are not shown. ${ }^{26}$

Although the authors observed high efficiency and low crosstalk, there are a few major disadvantages. First, the bandwidth of the waveguide crossing did not exceed $60 \mathrm{~nm}$. At a bandwidth of this size, this crossing is effective at a few wavelengths. This limited crossing performance restricts the wavelengths available for the entire photonic network. A wider bandwidth of at least $80 \mathrm{~nm}$ would retain performance across a larger range of wavelengths available for computation as other groups have demonstrated ${ }^{29,30}$. Second, the optimized structure of $70 \mu \mathrm{m}$ is too long for high-density photonic networks. ${ }^{31,32,33}$ Finally, a large percentage (38\%) of the total power transmitted through the crossing is not in the fundamental waveguide mode, but is instead lost through the higher order waveguide modes and radiated modes. These extra modes can interfere constructively or destructively. The various interference patterns introduce yet more variables for future further optimization of the waveguide structures. These issues are compounded by each subsequent crossings, affecting the performance of an entire circuit.

Another high-performing SOI multilayer waveguide crossing design was designed by the Bogaerts group achieved $97.5 \%$ transmission and $-40 \mathrm{~dB}$ crosstalk $^{27}$. The au- 
thors were able to achieve these results by combining wider intersecting waveguides with parabolic modal expanders along with a simple waveguide crossing. This improved the crossing transmission by quenching the propagating mode's wide-angle components from a ridge waveguide into a slab waveguide mode.

In their study, the authors compared simulated four separate crossings of two waveguides: a plain waveguide intersection, a waveguide crossing with one parabolic modal expander, a waveguide crossing with two parabolic modal expanders, and their waveguide crossing with silicon wire waveguides atop two parabolic modal expanders. These four cases are outlined in Figure I.4. For the plain waveguide intersection, the transmission was $67.6 \%$. In the waveguide crossing with just one parabolic modal expander, even more of the mode is diffracted out of the intended waveguide due to the longer length of the unconfined crossing area. Only $61.6 \%$ of the waveguide mode is transmitted through the crossing. This effect on the mode is made even more dramatic with modal expanders on both the incoming and unintentional waveguides, decreasing the transmission to $31.9 \%$. Bogaerts' waveguide crossing made of silicon wires atop two parabolic modal expanders increased transmission to $98.6 \%$ by increasing modal confinement along the crossing while gradually decreasing the refractive index width-wise in the waveguide leading up to the crossing.

There are several reasons for increased transmission with this compound structure. They achieved this compound structure by combining two parabolic mode expanders and a pair of orthogonal silicon wires, making what is known as a multimode interferometer. This multimode inteferometer utilized wires to provide a high enough refractive index contrast, preserving a high degree of modal confinement. The parabolic mode expanders decreased lateral refractive indices by changing the modal wavefronts from a curve to a straight line. This new wavefront interrupted the easily diffracted wide-angle modal components.

Although the authors were able to experimentally achieve $97.5 \%$ transmission and $-40 \mathrm{~dB}$ crosstalk, there is a major drawback with this approach. The fabrication of this device is fairly complicated and would be hard to incorporate into current chip assembly. 

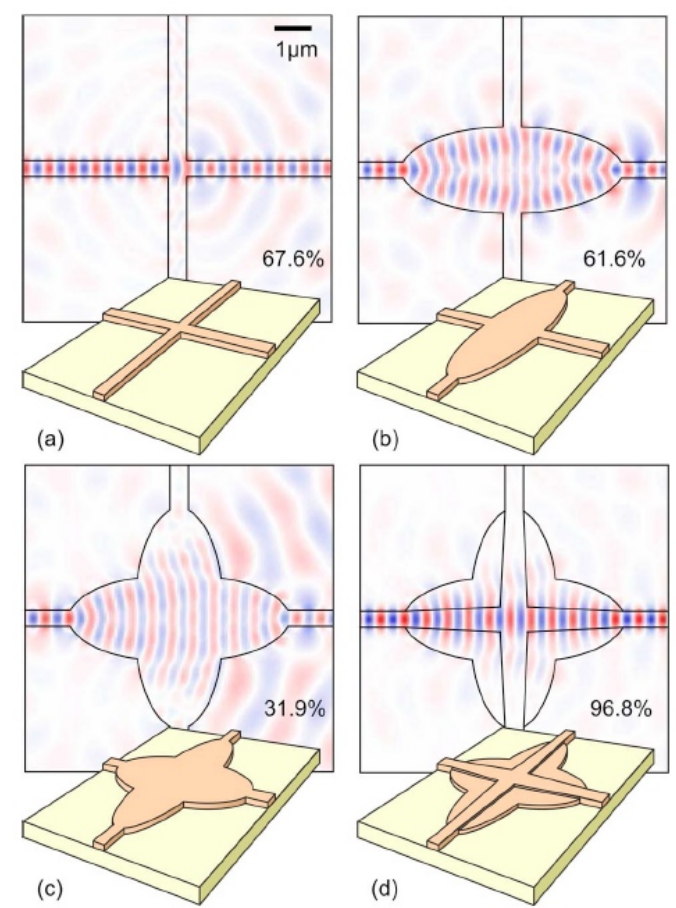

Figure I.4: Various Crossings with Simulated Transmission Efficiencies ${ }^{27}$

Multistep 248nm UV lithography and multiple dry etching steps shown in Figure I.5 are more complicated to incorporate into a photonic chip assembly than single-step processes. The first step is to deeply etch the outline and background of the waveguide crossings with a optical photolithography and deep reactive ion etch. The second step is to fabricate the parabolic expanders and isolate the wire waveguides with optical photolithography and a shallow reactive ion etch. The accuracy of the alignment between the two processes drastically affects the performance of this type crossings.

In 2010, a group led by Xiudong Sun designed waveguide crossings with perfect impedance matched, negative refractive index metamaterials ${ }^{28}$. By controlling the permittivity and permeability of the metamaterial crossing, the index of refraction can be negative. This negative index metamaterial can be designed to decrease crosstalk into unintended waveguides, decrease the size of the active waveguide crossing to the actual physical crossing, and increase the transmission through intended waveguides. Waveguide crosstalk originates at the crossing intersection from modal diffraction between the original 


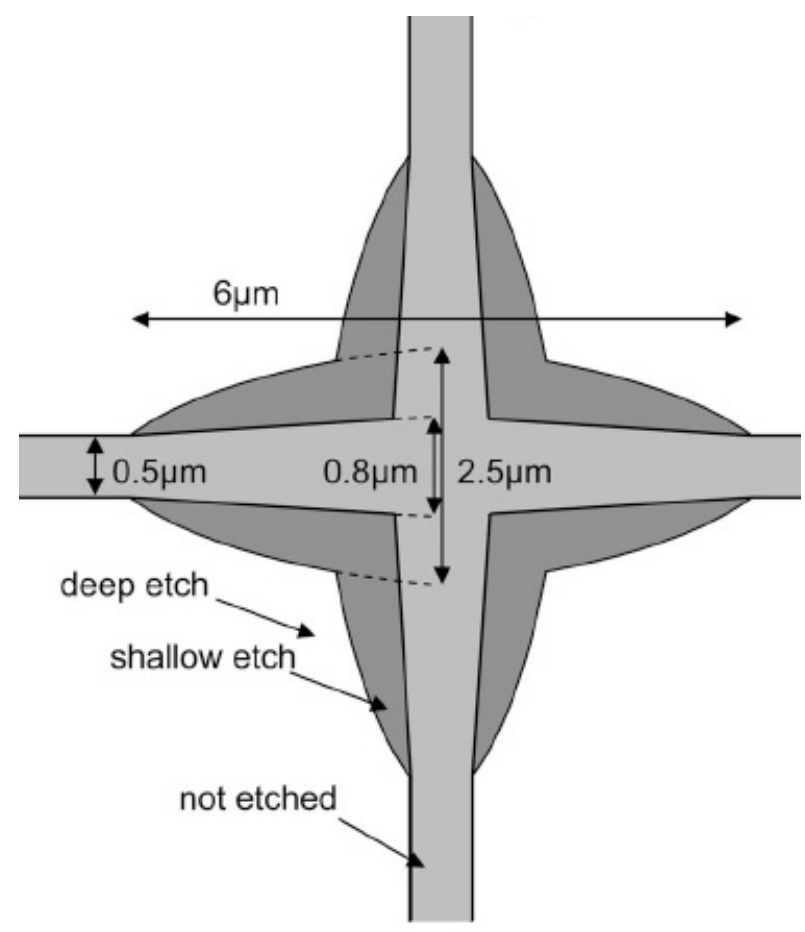

Figure I.5: Schematic of Proposed Bogaert's Waveguide Crossing 27

and unintended waveguide. While the crosstalk affecting transmission through the crossing region is inevitable, it can be mitigated by the crossing angle between the waveguides involved. As the crossing angle decreases to a particular point, the diffraction effect and crosstalk decreased.

The most common crossing for a single mode waveguide is a local long tapered mode expander crossing ${ }^{24,28}$. This type suppresses cross-diffraction by locally increasing the effective width crossing region as discussed previously ${ }^{28}$. The mode expander has performed well both theoretically and experimentally but is not ideal for highly integrated optical systems. Its major drawback is its large size $(>40 \mu \mathrm{m})$ and complicated shape optimizations. One example of such intensive shape optimization would be the evolutionary design progression. Metamaterial-based crossings along with small diffraction angle optimization has a marked advantage over this conventional approach. By optimizing only two parameters of the refractive index inside of the crossing and the angle of intersection, one can achieve similar performance metrics as shown in Figure I.6 and Figure I.7. A 
large refractive index in the crossing $n_{c}$ decreases the wavelength inside of the crossing $\lambda_{c}$ (which equals $\lambda_{o} / n_{c}$ ). However, with a large positive refractive index, there is a large impedance mismatch, resulting in large reflection off of the crossing interface into the environment. This problem can be solved by implementing a large negative absolute value $\left|n_{c}\right|$ refractive index material as the crossing. This means that even in the case of slight implementations from the original design, any light incident at this interface would reflect into the smaller refractive index, which in this case is the crossing. Theoretically, as the diffraction angle between the two waveguides $\theta$ progresses towards zero, the crosstalk is suprressed, yielding a reflection of zero due to perfect impedance matching.

Dr. Sun and his group demonstrated this principle by sminulating a SOI waveguide crossing at the frequency of $1.5 \mu \mathrm{m}$ at different angles and with different negative refractive index profiles ${ }^{28}$. The authors conjectured that this crossing design can be realized with fabrication techniques available in the literature. While this metamaterial crossing is possible to create, it would add a degree of difficulty to implement on an integrated optical circuit with other components fabricated with traditional SOI CMOS techniques.

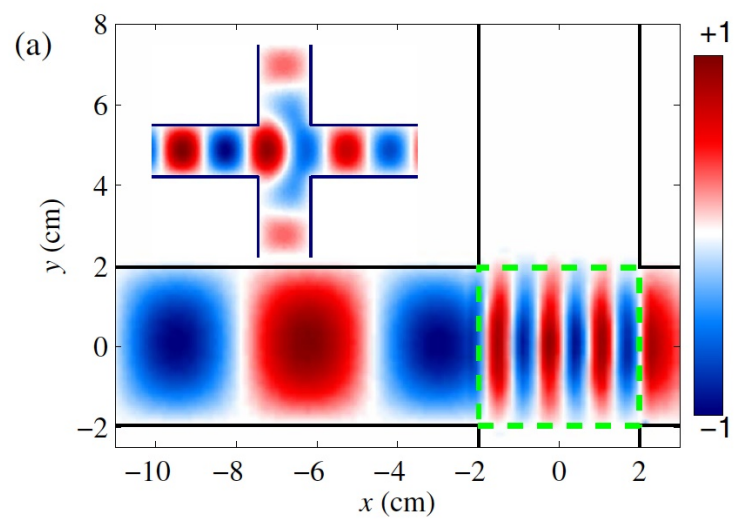

Figure I.6: 90 Degree Waveguide Crossings With and Without (Inset) Metamaterial Design. 28

With that being said, the authors' results are impressive and are on the order of required waveguide crossing performance metrics for use in integrated optics. With optimized metmaterial parameters and crossing angles, his group was able to demonstrate a 


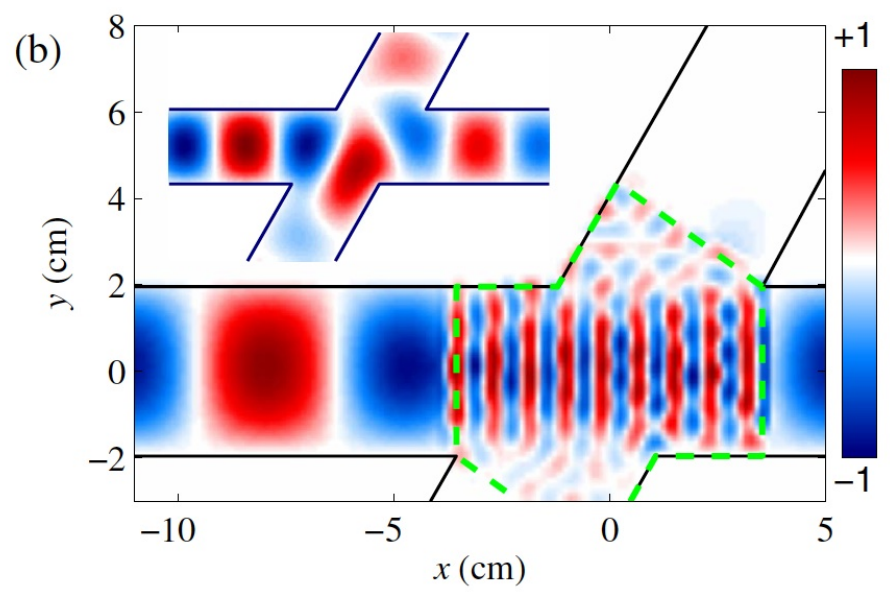

Figure I.7: 60 Degree Waveguide Crossings With and Without (Inset) Metamaterial Design. 28

transmission of more than 99 percent and a crosstalk of less than 0.5 percent as shown in Figure I. 8 below. ${ }^{28}$

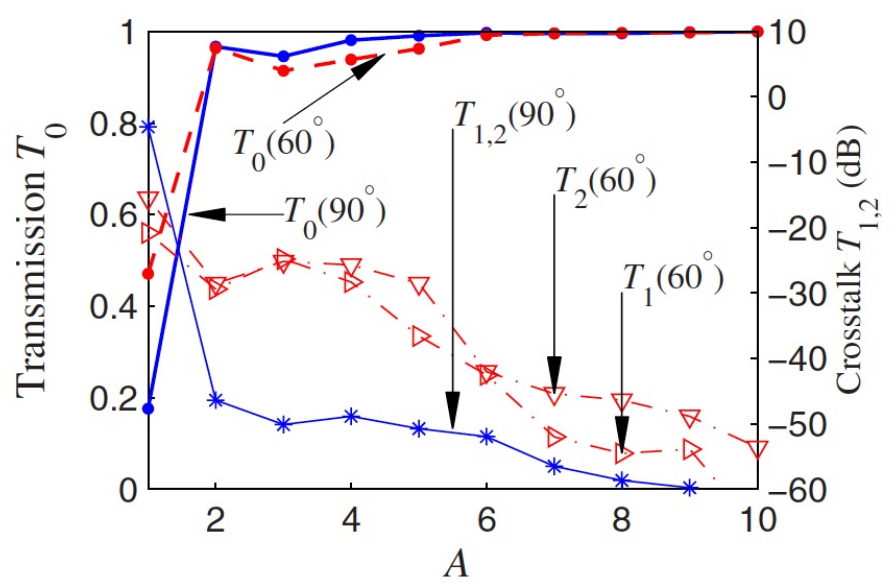

Figure I.8: Simulated Transmission Efficiencies and Crosstalk Loss for Various Negative Index Metamaterial Crossing Angles. ${ }^{28}$

In this section, we discussed four separate optical waveguide crossing designs: a periodic dielectric waveguide crossing, a silicon wire waveguide crossing utilizing adiabatic coupling, crossing utilizing parabolic wave expanders, and an impedance matched negative refractive index material waveguide crossing. For each waveguide crossing, we reviewed the related advantages and disadvantages. In Chapter 2, we will highlight the advantages 
of the Maxwell fisheye waveguide crossing over those in this section. The Maxwell fisheye waveguide crossing design and the realization through tapered waveguides designed with effective waveguide theory is discussed. Next, the 2D and 3D modeling performance of the crossing is evaluated along with results in Chapter 3. Then, both grayscale focused ion-beam lithography and grayscale electron-beam lithography procedures for device fabrication are examined in Chapter 4. Finally, in Chapter 5, future directions for this research is articulated. 


\section{CHAPTER II}

\section{Proposed Maxwell Fisheye Waveguide Crossing Design}

In this section, we will discuss the basics of the Maxwell fisheye and derive the MFE profile with both ray optics and transformation optics. Here we discuss a new type of waveguide crossing designed to retain high transmission efficiency in a small crossing area while crossing several waveguides simultaneously ${ }^{34}$. A major advantage of the MFE profile as a waveguide crossing is that it focuses an image source from one lens edge to the

opposite with no loss or aberration ${ }^{35,36,37}$. This low loss and low aberration mitigates any crosstalk into unintended waveguides. In addition to this lower crosstalk, we hypothesize that the transmission of this waveguide crossing will be high due to the perfect imaging of the source on the edge of the waveguide crossing. Furthermore, we hypothesize this radial refractive index profile can realized by modulating the thickness of a tapered SOI waveguide system which in turn modulates the modal refractive index.

The purpose of this study is to propose a solution to the waveguide crossing problem by merging a Maxwell fisheye index profile with tapered waveguides on an SOI architecure. The proposed crossing should produce a compact, efficient waveguide hub crossing that is able to be fabricated with straightforward CMOS fabrication techniques.

\section{II.1 Background of Maxwell Fisheye Lens}

In 1854, Maxwell proposed a lens where light is focused onto the opposite side with no aberration as shown in the figure below ${ }^{35}$. The refractive index profile of this lens is described as

$$
n=\frac{n_{o}}{1+\left(\frac{r}{R}\right)^{2}}
$$

where $n_{o}$ is the refractive index in the lens center, $\mathrm{r}$ is the radial axis, and $\mathrm{R}$ is the lens 
radius. An example of a Maxwell fisheye refractive index profile is demostrated in Figure II. 1 below with the highest refractive index of 3.2 represented by the color red and the lowest refractive index of 1.6 represented by the color blue.

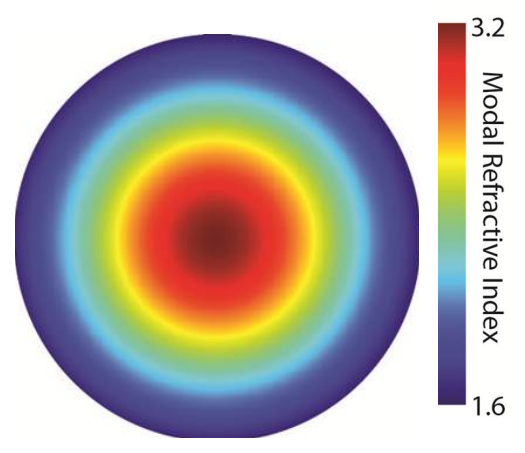

Figure II.1: An Example of the Maxwell Fisheye Lens Refractive Index Profile

In 1854, Maxwell derived this lens with ray optics theory ${ }^{35}$. However, the ray optics paradigm has a major disadvantage ${ }^{38}$. It does not take into account the wave nature of light. With ray optics, the size of the lens would not severely affect performance, but this is not experimentally the case. The wave nature becomes more relevant as the size of the lens decreases to the order of the wavelength of light. This wave nature is important for our lens size and its effect can be verified experimentally. To include the wave nature of light in this refractive index profile, we describe this lens with transformation optics. In transformation optics, optical properties in a transformed physical medium are spatially manipulated to guide light along intended propagation directions given in a virtual medium. Specifically for a Maxwell fisheye, the curved space in the transformed physical space of the lens is mapped as a virtual flat straight propogation direction.

\section{II.2 Derivation of Maxwell Fisheye Lens Refractive Index Profile}

\section{II.2.1 Ray Optics Approach}

In order to describe how light travels in gradient refractive index materials with the ray optics approach, we must consider the fact that light rays travel the path with the smallest amount of time between two points. In homogenous refractive index materials, this path 
would be a straight line. In heterogenous refractive index materials, this is not the case. In such a material, light takes the shortest optical path, which is a curved path as shown in Figure II. 2 below. The light rays travel in the various refractive indices at different speeds. The dotted line represents the straight line optical path in a homogenous refractive index profile. The solid line represents the curved line optical path in a heterogenous refractive index.

This curving of the path in a heterogenous refractive index profile is known as Fermat's

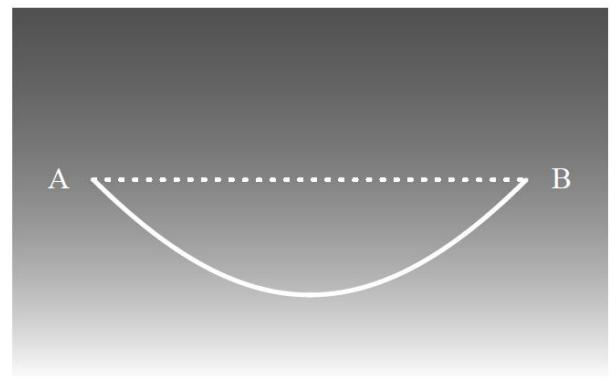

Figure II.2: Fermat's Principle of Least Action in heterogenous refractive index materials. $^{39}$

Principle of Least Action and is mathematically described by the following equation:

$$
s=\int_{a}^{b} n d l=\int_{a}^{b} n \sqrt{d x^{2}+d y^{2}+d z^{2}}
$$

where $x, y$, and $z$ are the real space coordinates between points $a$ and $b$, and $n$ is an arbitrary spatially varying refractive index.

\section{II.2.2 Transformation Optics Approach}

One way to describe the Maxwell fisheye refractive index profile through the transformation optics paradigm is through mapping the curved surface as a flat surface. First, we start with the stereographic projection of a 3D sphere with radius $r_{o}$ along the $2 \mathrm{D} \mathrm{x}, \mathrm{y}$ plane as a great circle. In the physical space, light propogates along the great circle, or the geodesic of the sphere. In this system, the source is perfectly imaged on the antipodal point along the geodesics of the lens sphere via a specific non-uniform refractive index profile. 
The coordinates of the original physical circular system $(x, y, z)$ in terms of the transformed flat system $\left(x^{\prime}, y^{\prime}, z^{\prime}\right)$ are

$$
\begin{array}{r}
x=\frac{x^{\prime}}{1+z^{\prime} / r_{o}} \\
y=\frac{y^{\prime}}{1+z^{\prime} / r_{o}} \\
r_{o}^{2}=x^{\prime 2}+y^{\prime 2}+z^{\prime 2}
\end{array}
$$

The coordinates of the flat transformed system $\left(x^{\prime}, y^{\prime}, z^{\prime}\right)$ in terms of the physical circular system $(x, y, z)$ are shown in the equations below:

$$
\begin{array}{r}
x^{\prime}=\frac{2 x}{1+\left(r / r_{o}\right)^{2}}, \\
y^{\prime}=\frac{2 y}{1+\left(r / r_{o}\right)^{2}}, \\
z^{\prime}=r_{o} \frac{\left(r / r_{o}\right)^{2}-1}{\left(r / r_{o}\right)^{2}+1}, \\
\text { and } \\
r^{2}=x^{2}+y^{2}
\end{array}
$$

As light propogates along the circular system, the line element $(d s)$ in flat transformed space with homogenous refractive index of $n_{o}$ is

$$
d s^{2}=n_{o}^{2}\left(d x^{\prime 2}+d y^{\prime 2}+d z^{\prime 2}\right)
$$

To enact this change in physical space, the spatial refractive index profile is reflected in a transformed coordinate system as shown in Figure II.3 shown below. In terms of the physical coordinates $x, y$, and $r_{o}$, the line element $(d s)$ and the refractive index profile $(n)$ is described as

$$
d s^{2}=n^{2}\left(d x^{2}+d y^{2}\right)
$$




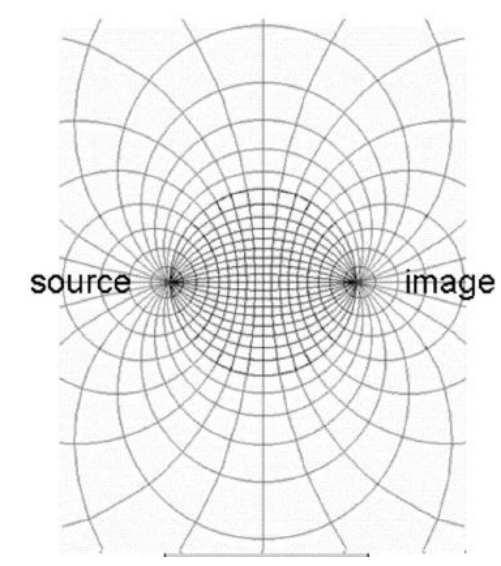

Figure II.3: Optical transformation of the physical space Maxwell fisheye lens imaging. ${ }^{40}$

and

$$
n=\frac{2 n_{o} r_{o}^{2}}{x^{2}+y^{2}+r_{o}^{2}}
$$

The Maxwell fisheye lens profile works as designed for wavelengths much smaller than the radius of the lens; however, as the wavelength increases, the limitations of this design become more apparent. When the wavelength of incident light $(\lambda)$ is on the order of lens radius, the resolution of the lens decreases to $\frac{\lambda}{3}$ for transverse magnetic polarized light and $\frac{\lambda}{4}$ to as large as $\frac{\lambda}{2}$ for transverse electric polarized light ${ }^{39}$.

In this section, we reviewed the basics of the Maxwell fisheye and derived the MFE profile with both ray optics and transformation optics. Our group proposed a new type of waveguide crossing to retain high transmission efficiency in a small crossing area while crossing several waveguides simultaneously ${ }^{34}$. A major advantage of the MFE profile as a waveguide crossing is that it focuses an image source from one lens edge to the opposite with no loss or aberration $35,36,37$. This low loss and low aberration mitigates any crosstalk into unintended waveguides. In addition to this lower crosstalk, we hypothesized that the transmission of this waveguide crossing will be high due to the perfect imaging of the source on the edge of the waveguide crossing. Furthermore, we hypothesized that this 
radial refractive index profile can realized by modulating the thickness of a tapered SOI waveguide system which in turn modulates the modal refractive index. In the following section, we will discuss the performance of the Maxwell fisheye waveguide crossing both in $2 \mathrm{D}$ and $3 \mathrm{D}$ simulations. 


\section{CHAPTER III}

\section{Modeling and Performance}

To compare our design of a Maxwell fisheye crossing performance to other crossings reported in the literature, we employ both 2D and 3D finite element analysis method with commercial software COMSOL ${ }^{34}$. In this section, we will discuss the details of these simulation. For the 2D simuation, we will review input field parameters, output field parameters, and waveguide crossing performance in terms of crosstalk and transmission efficiency. For the $3 \mathrm{D}$ simulation, these three topics will also be discussed.Next, the results from both types of simulation will be compared. Afterwards, the discrepancies between the two simulation results will be examined with a projection for future study.

\section{III.1 2D Simulation of MFE Crossing Performance}

For the 2D simulations, we eliminated reflections at the boundaries by surrounding them with perfectly matched layers (PMLs) ${ }^{34}$. The schematics for $2 \mathrm{D}$ simulated crossings of both two and multiple waveguides are shown in the Figures III.1 and III.2 below, respectively. In Figure III.1, two waveguides were crossed orthogonally with a $6 \mu \mathrm{m} \mathrm{MFE}$ waveguide crossing at the intersection. In Figure III.2, seven equidistant waveguide were crossed orthogonally with a $6 \mu \mathrm{m}$ MFE waveguide crossing at the intersection. In both figures, the red and blue represents the crest and trough of the mode propogating through the waveguide, respectively. For both cases, the center left waveguide was excited with an electric field profile of the form of a Gaussian wave given by the following equation:

$$
E=a e^{\frac{-(x-b)^{2}}{2 c^{2}}}
$$

where $a$ is the amplitude of the input wave, $b$ is the center of the input wave, and $c$ is the constant scaling factor, which is related to the full width half maximum (FWHM). This 
scaling factor $c$ is given by the following equation:

$$
c=\frac{\mathrm{FWHM}}{2 \sqrt{2 \ln (2)}}
$$

For our simulations, $a$ was chosen to be $1 \mathrm{~V} / \mathrm{m}, b$ was the center of the waveguide or $0 \mathrm{~nm}$, and the FWHM was chosen to be $50 \mathrm{~nm}$.

To calculate the transmitted power efficiencies of each waveguide setup, the fields were captured both 2 microns before the lens and 2 microns after the lens on the intended waveguide. The powers at these positions were compared and multiplied by $100 \%$. To calculate the crosstalk of the signal into the unintended waveguides for the two waveguide crossing case, the fields were captured 2 microns before the lens in the intended waveguide and 2 microns after the lens in both of the unintended waveguides.

For our 2D study, both the crossing and the waveguides were designed for TM polarization, yielding $E_{z}, H_{x}$ and $H_{y}$. To maintain a modal index of 1.6 in the 2D model, we utilized waveguides with a refractive index of 3.5 and a width of $53.4 \mathrm{~nm}$. To prevent reflection at the intersection of the waveguide and the waveguide crossing due to an impedance mismatch, the index at the edge of the crossing was chosen to match the waveguide modal index of 1.6. This yielded a maximum modal index at the center of the crossing to be 3.2. The wavelength of interest was 1.3 microns. To simulate the lens on an SOI architecture, the refractive index of the background was chosen to match that of the silicon dioxide layer $(n=1.5)$ in the SOI system. For the section of the 2D study involving crossing multiple waveguides, the schematic is different. Three to eight equidistant waveguides were added.

As a result of this $2 \mathrm{D}$ study with only two waveguides with the Maxwell fisheye crossing, the transmission efficiency and crosstalk was found to be $97.2 \%$ and $2 \%$, respectively. The rest of the signal $(0.8 \%)$ was lost to the environment through insertion losses. The electric field profile of this case is shown in Figure III.1 below. In this case, limited bandwidth operation was observed. This was not attributed to the waveguide crossing it- 


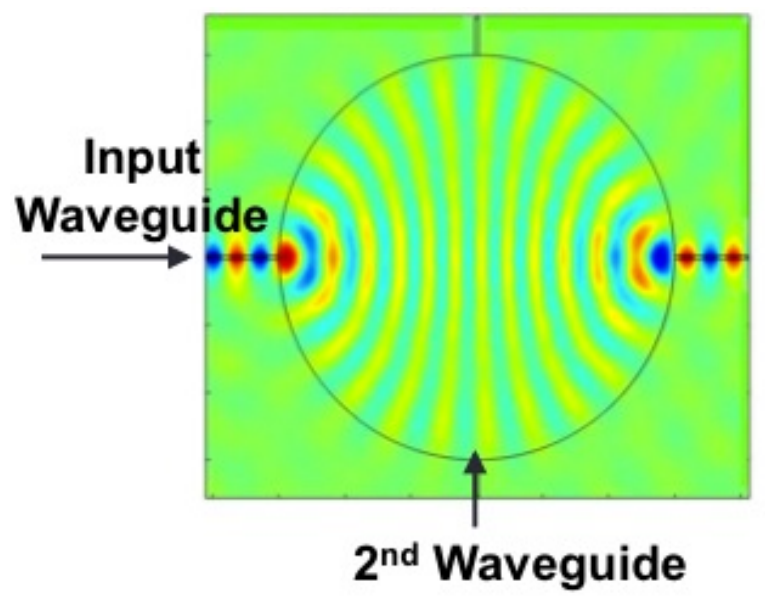

Figure III.1: Electric Field Inside of a $6 \mu \mathrm{m}$ Wide Maxwell Fish Eye Crossing with One Auxillary Waveguide ${ }^{34}$

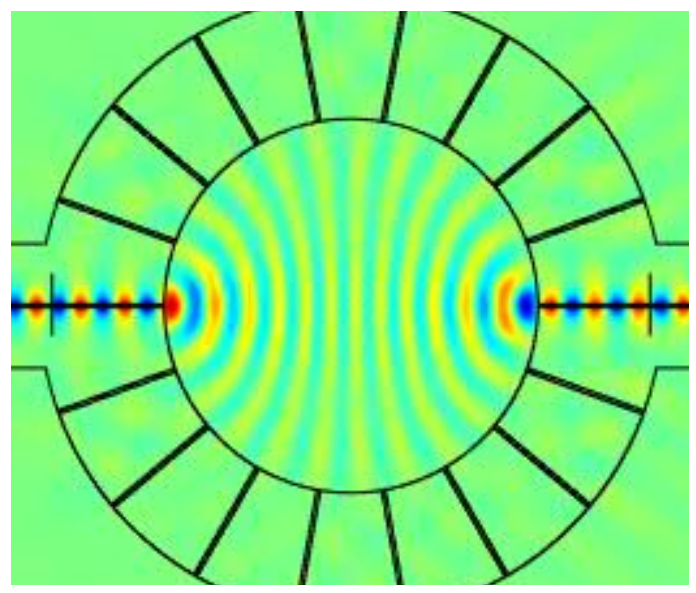

Figure III.2: Electric Field Inside of a $6 \mu \mathrm{m}$ Wide Maxwell Fish Eye Crossing with Eight Auxillary Waveguides

self but to the limited broadband modal operation of the incoming waveguide. The 2D Maxwell fisheye refractive index profile behaved as a waveguide crossing excited with a dipole on the crossing edge performed for a broad bandwidth up to R/3. This R/3 impediment was due to the fact that the wavefront was too large to respond to sudden changes in the refractive index profile.

The transmission efficiencies with multiple crossing waveguides is presented in Figure III.3 below. While the $6 \mu \mathrm{m}$ wide crossing coupled the majority of the incident signal into its intended waveguide at the wavelength of $1.3 \mu \mathrm{m}$, there is a threshold of effective- 
ness that was crossed with eight equidistant waveguides for a $6 \mu \mathrm{m}$ wide MFE crossing. When eight waveguides enter the Maxwell fisheye crossing of this size, the modal electric field of the incoming waveguide began to couple into the surrounding waveguides, diluting the point source. This dilute point source entered the crossing, which was designed for a focused point source. Subsequently, the performance was diminished.

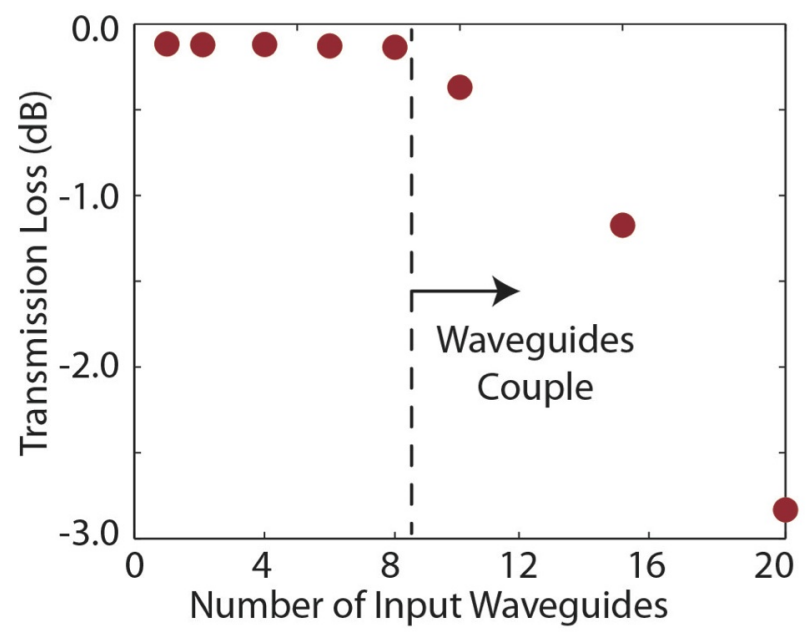

Figure III.3: Transmission Loss Plot of Maxwell Fish Eye Crossing with Up to Seven Equidistant Waveguides

\section{III.2 3D Simulation of MFE Crossing Performance}

For 3D simulations, the Maxwell fisheye refractive index profile was implemented by modulating the thickness of silicon in a TE air-SOI asymmetric slab waveguide system as shown in Figure III.4 below. With the TE air-SOI asymmetric waveguide slab system, we relate the modal refractive index directly with waveguide height in nanometers as shown in Figure III.5. For more detail on the TE asymmetric waveguide modal index relation we utilized, please refer to Appendix A. With this relation, we were able to produce the actual radially symmetric height needed to implement the refractive index for the MFE waveguide crossing as shown in Figure III.6. This 2D height profile was rotated to create a 3D radially symmetric profile as shown in Figure III.7 This 3D height profile was imported into the commercial finite element analysis method software COMSOL for testing. The incoming 
waveguides for the 3D study were ridge waveguides with dimensions of $250 \mathrm{~nm}$ tall and $250 \mathrm{~nm}$ wide. To ensure a single-mode operating waveguide, we employed the operating wavelength used for experimental realization, $1.2 \mu \mathrm{m}$.

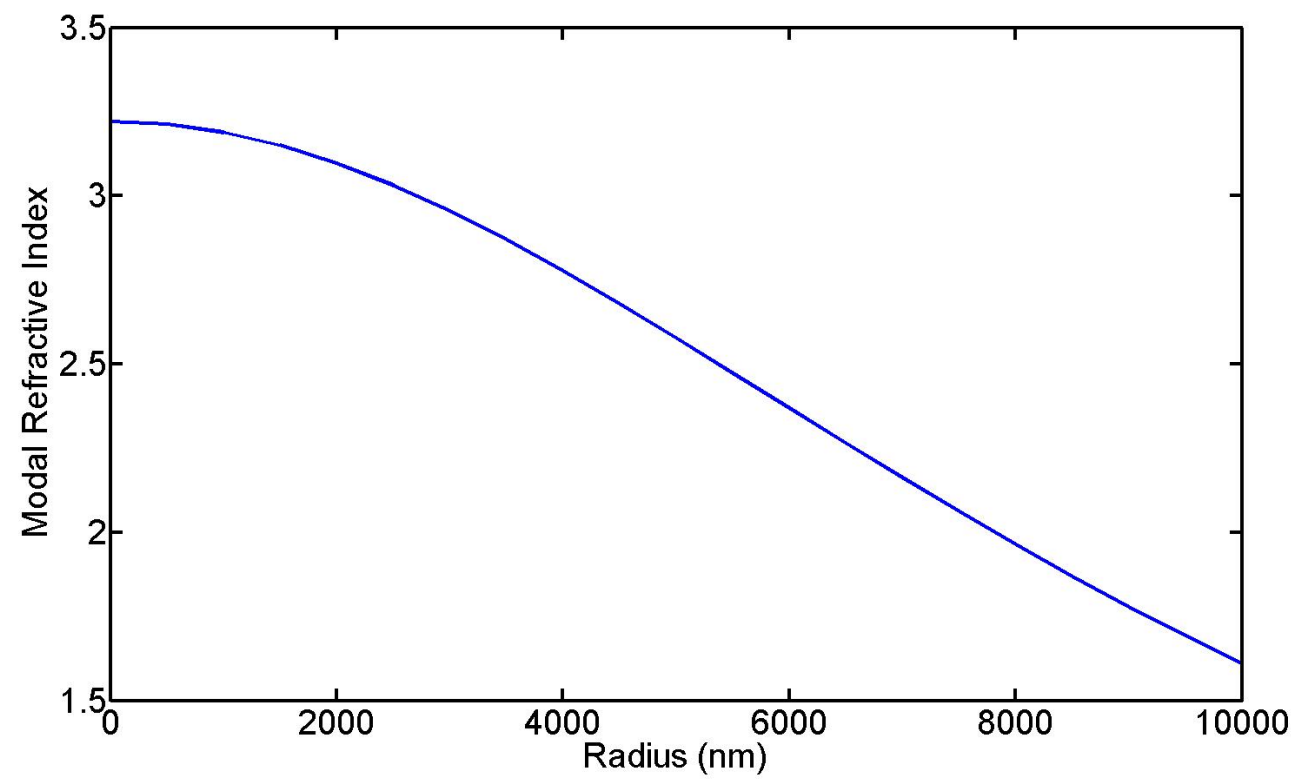

Figure III.4: Modal Refractive Index Profile of a $10 \mu \mathrm{m}$ Radius Maxwell Fish Eye

In order to eliminate reflections at all boundaries, we surrounded them with $3 \mathrm{D}$ domains with boundary conditions of perfectly matched layers (PMLs). The schematics for 3D simulated crossings is shown in the figure below. The input electric field for the 3D ridge waveguide was of the form of a Gaussian wave given by:

$$
E=A e^{-\left(\frac{\left(x-x_{0}\right)^{2}}{2 c_{x}^{2}}+\frac{\left(y-y_{o}\right)^{2}}{2 c_{y}^{2}}\right)},
$$

where $A$ is the amplitude of the input wave, $x_{o}$ is the center of the input wave on the $\mathrm{x}$ axis, $y_{o}$ is the center of the input wave on the y-axis, and $c_{x}$ and $c_{y}$ are the constant scaling factors, which is related to the full width half maximum (FWHM) on the $\mathrm{x}$ and $\mathrm{y}$ axes, 


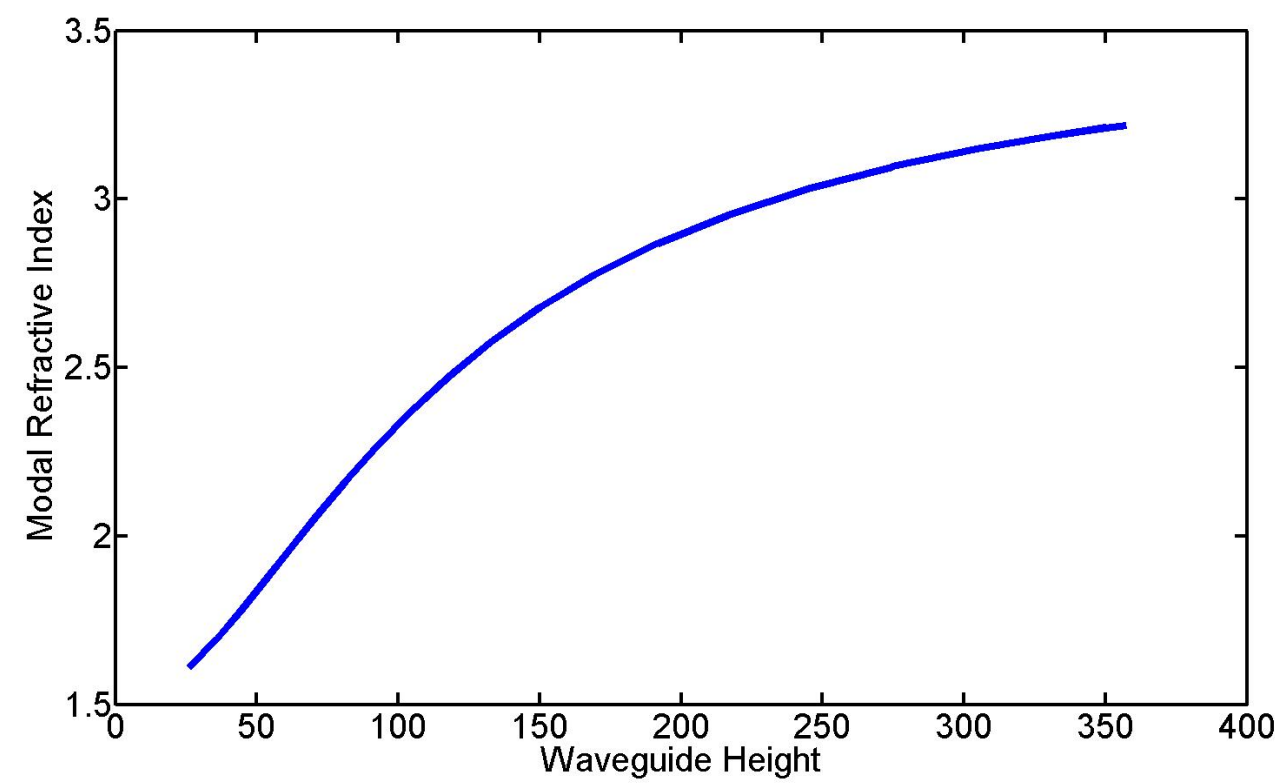

Figure III.5: 3D Maxwell Fish Eye Modal Refractive Index With Respect to Tapered Slab Waveguide Height

respectively. This scaling factor $c$ is given by the following equation:

$$
\begin{aligned}
& c_{x}=\frac{\mathrm{FWHM}_{x}}{2 \sqrt{2 * \ln (2)}} \\
& c_{y}=\frac{\mathrm{FWHM}_{y}}{2 \sqrt{2 * \ln (2)}}
\end{aligned}
$$

For our simulations, $A$ was chosen to be $1 \mathrm{~V} / \mathrm{m}, b$ was the center of the waveguide or $0 \mathrm{~nm}$, $F W H M_{x}$ was chosen to be $150 \mathrm{~nm}$ and $F W H M_{y}$ was chosen to be $200 \mathrm{~nm}$. This produced a mode that was both TE- and TM-like, unlike the pure TM mode for the 2D simulations.

Another thing to note is that in order to efficiently use available computing power, the 3D study was restricted to observing the transmission efficiently and crosstalk of only 2 crossed waveguides. To calculate the transmitted power efficiencies of each waveguide setup, the fields were captured both 2 microns before the lens and 2 microns after the lens on the intended waveguide. To calculate the crosstalk of the signal into the unintended waveguides for the 2 waveguide crossing case, the fields were captured 2 microns before 


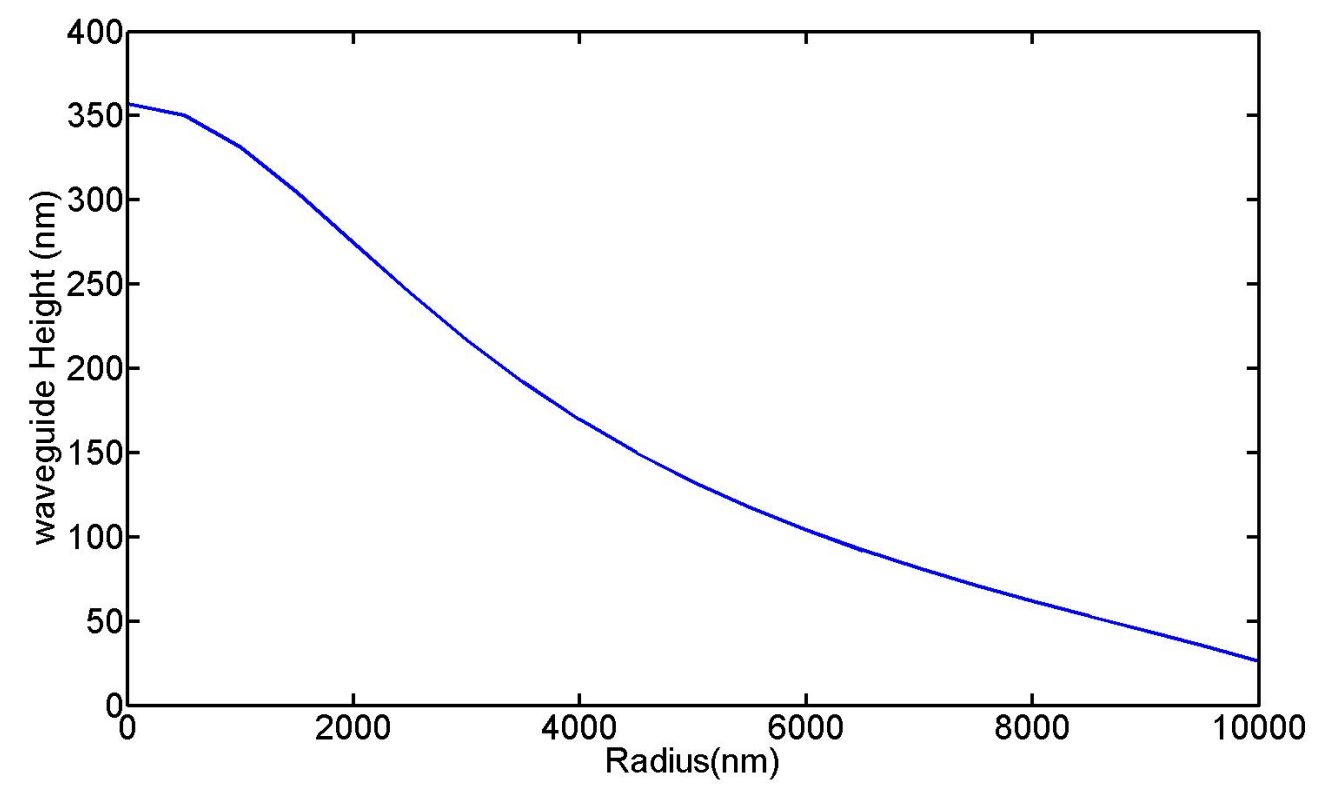

Figure III.6: Waveguide Height (in nm) for a $10 \mu$ m Radius Maxwell Fish Eye

the lens in the intended waveguide and 2 microns after the lens in both of the unintended waveguides.

The transmission efficiency of the 3D simulated waveguide crossing was found to be $72 \%$. The majority of the scattered light was at the intersection of the ridge waveguide and the waveguide crossing. One possible explanation for this is that the crossing height was derived with TE slab parameters, but the ridge waveguide mode contained both TE and TM elements. At the intersection, the TE-like component's modal index of 1.6 matched the TE height profile modal index of 1.6. However, for the TM-like ridge waveguide field component encountered a lower slab modal index at the edge of the lens. This is due to the fact that the height of the TE slab waveguide yields a lower modal index than a TM slab waveguide. This impedance mismatch that the TM-like component of the ridge waveguide mode encounters contributes to large reflections seen at the intersection of the crossing. Subsequently, the performance is diminished. To confirm this result, more 3D studies with various types of incoming waveguides should be performed.

To compare our design of a Maxwell fisheye crossing performance to other cross- 


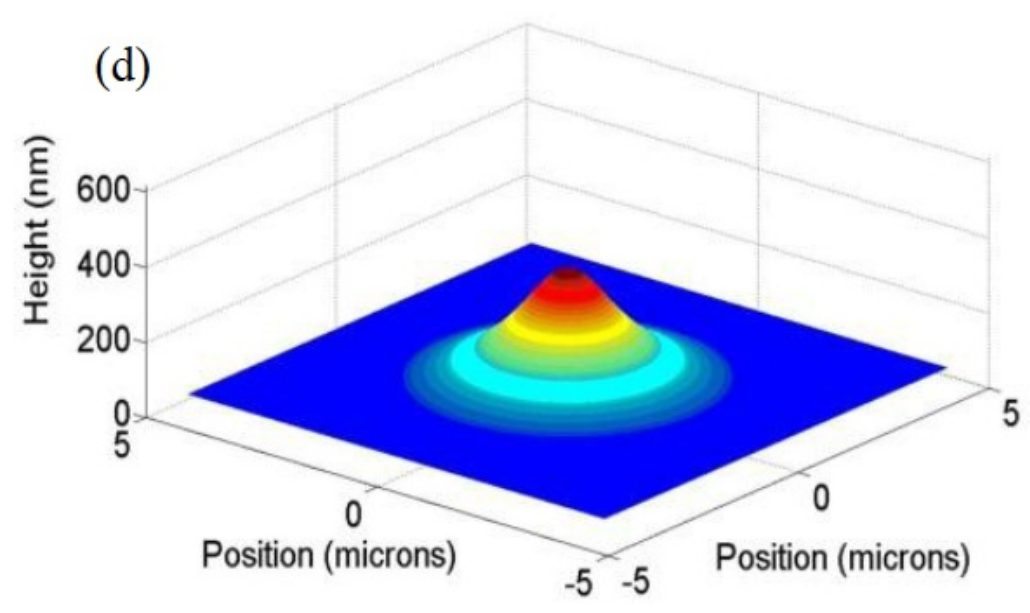

Figure III.7: 3D Maxwell Fish Eye Height Profile Realized By TE Tapered Slab Waveguides

ings reported in the literature, we employed both 2D and 3D finite element analysis method with commercial software COMSOL. In this section, we discussed the details of these simulation. For the 2D simuation, we reviewed input field parameters, output field parameters, and waveguide crossing performance in terms of crosstalk and transmission efficiency. For the 3D simulation, these three topics were also discussed. In addition, we reviewed the height profile determined by effective waveguide theory. Next, the results from both types of simulation were compared. Afterwards, the discrepancies between the two simulation results were examined with a projection for future study. 


\section{CHAPTER IV}

\section{Fabrication Protocols}

In this chapter, we will discuss the conversion of design height to actual device height via bitmap translation. The resulting bitmaps focused ion beam (FIB) and electron-beam lithography (EBL). Both types of fabrication have their advantages and challenges, which we will discuss. Finally, we will discuss the agreement of final FIB fabrication to designed MFE waveguide crossing.

\section{IV.1 Grayscale Bitmap to Fabricated Device Height Translation}

A novel grayscale fabrication method was developed in 2005 using a computer-generated bitmap pattern with 256 gray levels ${ }^{41}$. This bitmap was then translated into FIB or EBL dose levels, making three-dimensional varied structures. This method increases the scope of milled materials from binary to arbitrary height profiles, perfect for our application. For our waveguide crossings, we constructed bitmap patterns through MATLAB programming converting desired height to corresponding gray levels. The final milled heights have units of nanometers, while the grayscale bitmap levels do not. The relationship between the desired milled height and the grayscale bitmap is given by, $\mathrm{G}_{i}=255 * D_{i} / D_{o}$, where $G_{i}$ is the desired grayscale bitmap level, $D_{i}$ is the desired milled depth (nm), and $D_{o}$ is the maximum milled depth (nm). This formula produces bitmap level between 0 (black) and 255 (white). The black bitmap level corresponds to maximum FIB exposure and mimimum milled height. The white bitmap level corresponds to mimimum exposure and maximum milled height.

For our design, the maximum relief height $\left(D_{o}=500 \mathrm{~nm}\right)$ is the height of the silicon layer in our SOI platform ${ }^{34}$. The total number of pixels used in our pattern was 810,000 for a 900 x 900 bitmap. Both our FEI-200 and RAITH-EBL was equipped with operation software that converted the grayscale between 0 and 255 into the corresponding dose required 


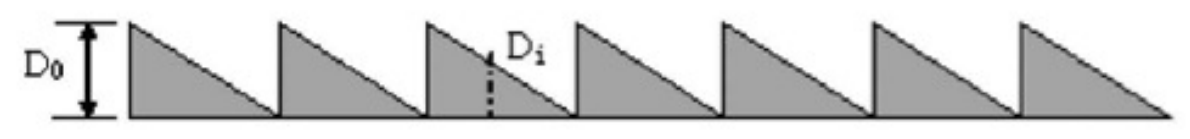

Figure IV.1: Grayscale Lithography with $D_{o}$ as the Largest Feature Height and $D_{i}$ as the Spatially Varying Feature Height ${ }^{41}$

for the desired milling depth.

\section{IV.2 Focused Ion Beam Grayscale Lithography Procedure}

The precision of the final device is dependent on milling parameters, such as beam current, overlapping, pixel spacing, ion energy, exposure area, and dwell time ${ }^{34}$. The ion beam spot size plays a direct role in the grayscale pattern resolution, making the selection of beam spot aperture, pixel spacing, and the chosen bitmap pattern size relevant to the dimensions of the final milled waveguide crossing. The resulting bitmap was uploaded onto our FEI-200 FIB for direct grayscale milling with $350 \mathrm{pA}$ - $1.0 \mathrm{nA}$ beam current, 50\% overlapping in $\mathrm{X}$ and $\mathrm{Y}$ directions, $60 \mathrm{~nm}$ pixel spacing and 30-keV Ga+ ion energy in an area of $20.0 \times 20.0 \mu \mathrm{m}^{2}$. The normally-oriented beam moves during the milling procedure with as fast of a raster as possible, which is dependent on the dwell time. On our machine, the lowest possible dwell time for each pixel is $0.1 \mu$ s. Figure IV.2 below is an example of the resolution we are able to achieve with bitmap grayscale FIB lithography in VINSE. 


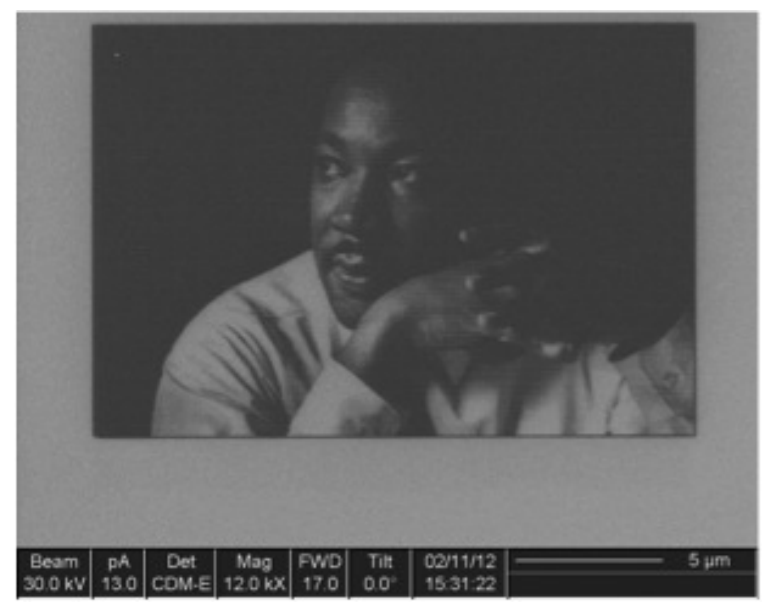

Figure IV.2: Example of Grayscale FIB Fabrication Resolution Capabilities in VINSE

An issue we discovered when milling the radially symmetric waveguide crossings was the presence of a surplus circle in the center of the crossing as shown in the Figure IV.3 below. Figure IV.3 is an AFM micrograph taken of our final FIB direct-milled MFE waveguide crossing on SOI, as well as an atomic force microscope (AFM from NanoScope) with tapping mode. This surplus circle is worth noting because any defect of this nature can negatively affect the focusing power of the lens and scatter light from the waveguide crossing into the environment. This circle happens due to the inability of the milling software to distinguish between small differences in dwell time in terms of the gray-scale pattern ${ }^{41}$. 


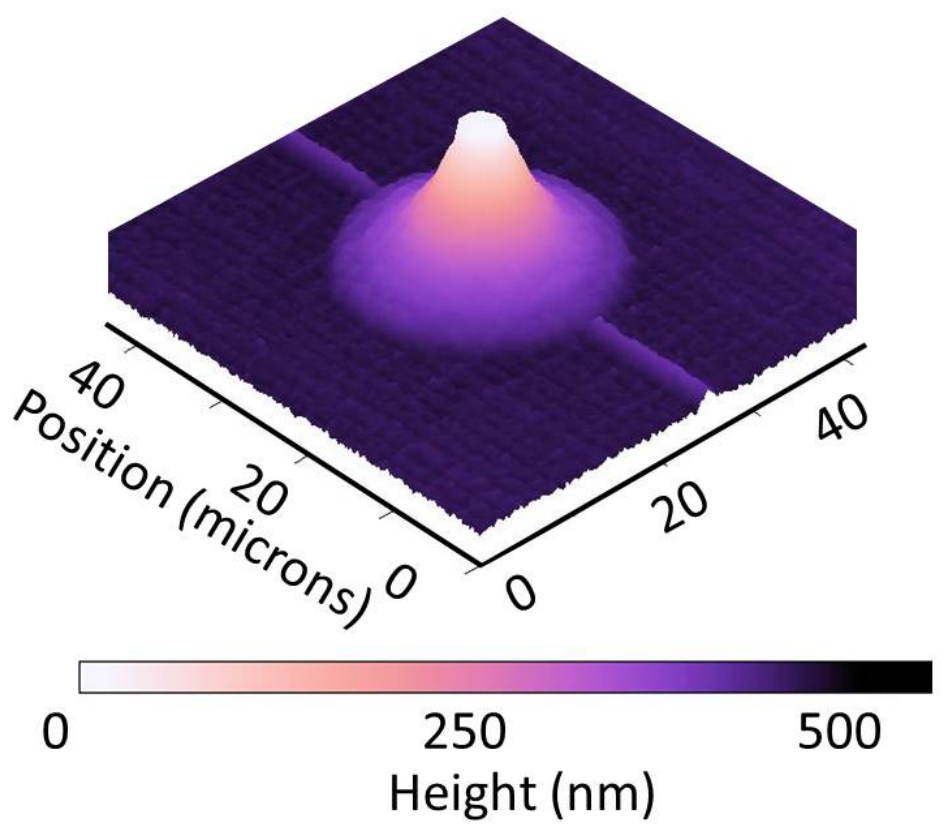

Figure IV.3: Grayscale FIB Fabrication of Maxwell Fisheye Waveguide Crossing 34

The diameters of these circular artifacts vary depending on the resulting gradient resolution $^{41,42}$. By decreasing the beam size and current, the resolution increases. Through increased resolution, one can negate these effects during milling. However, this introduces another issue. By decreasing the spot size and current, one not only increases resolution, but also write time. Due to increased write time, the element of stage drift more strongly affects the accuracy of pattern writing. It is a trade-off; one must balance high gradient resolution with a high degree of milling accuracy.

\section{IV.3 Grayscale Electron-Beam Lithography with RIE Pattern Transfer Procedure}

Grayscale electron-beam lithography is similar to grayscale FIB milling in that dot dose exposure locally controls the resultant device height ${ }^{34,43}$. Experimentally, the dot clearing dose was fitted to the resultant height for various bitmap spacings. With this information, any arbitrary grayscale bitmap was able to be converted to localized dot dose exposure patterns through a curve fitted function which results in arbitrary varying height profiles. 
However, there are several technical complications to avoid when using dot dose based grayscale lithography. The largest of these is the proximity effect. The proximity effect from electron scattering can decrease the accuracy of the exposed design with decreasing feature sizes, which can severely affect the performance of smaller diameter waveguide crossings $(10 \mu \mathrm{m})$. With small crossings and small pixel spacings, a pixel with a designated dose can affect the starting depth of a nearby pixel by pre-exposing it. This effect is known as blurring, and precisely controlling exposure in nearby dot pixel areas is essential to the fabrication accuracy of an intended design. To prevent this blurring, one can utilize patterns over a larger area along with greater pixel spacing. However, there is a limit. With large patterns, stitching between write areas can create jogs in fabricated grayscale patterns. These jogs function as a defect from which light inside of the waveguide crossing can scatter into the environment. Also, implementing large patterns puts a large computational load on the lithography software, causing it to crash often.

Silicon wafer samples were spin-coated with 50k chain length polymethylmethacrylate (PMMA) in anisole with $20 \%$ solid content at $4000 \mathrm{rpm}$ for $55 \mathrm{~s}$, resulting in a final photoresist thickness of $1100 \mathrm{~nm}$. Then, the samples were baked at $180^{\circ} \mathrm{C}$ for $8 \mathrm{~min}$. Ellipsometry and profilometry were used to measure the silicon and PMMA thicknesses. Patterns were exposed using a RAITH EBL operated at $30 \mathrm{keV}$. To develop the resist, the sample was immersed in 1:3 methylisobutyl ketone (MIBK): isopropyl alcohol (IPA) for $60 \mathrm{~s}$ and pure IPA for 60 seconds at room temperature.

After the PMMA is patterned according to the above EBL protocol, reactive ion etching was used to transfer the radial test pattern from the resist onto the silicon substrate, (i.e, the silicon was masked by the grayscale patterned resist) ${ }^{34,44}$ as shown in Figure IV.4 below. The grayscale bitmap pattern used in EBL patterning is shown in the top left of Figure IV.4. The second image in Figure IV.4 shows a cross section of the patterned PMMA on crystalline silicon on top of quartz. The third image in Figure IV.4 depicts the completed RIE transfer of the PMMA pattern into the silicon substrate underneath. Depending on 
the PMMA and silicon etch rate ratio, the silicon substrate was etched according to the varying resist thickness. RF power, ICP power, gas composition, and gas pressure were used to control the RIE etching accuracy of our designs. Then, the required optimization experiments and error analyses were performed. The active gases used for etching were $\mathrm{O}_{2}$ and $\mathrm{SF}_{6}$, where the $\mathrm{SF}_{6}$ flow rate was $25 \mathrm{sccm}$ and the $\mathrm{O}_{2}$ flow rate varied.

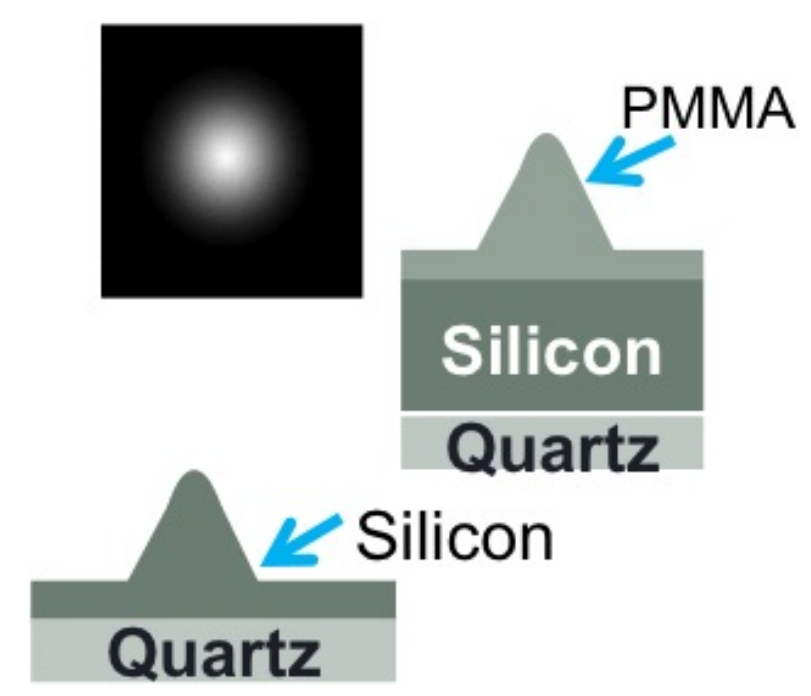

Figure IV.4: Grayscale EBL-RIE Protocol on an SOI Substrate

The resulting silicon and PMMA etching rates obtained through our optimization experiments are provided in the table below. It is evident that a connection exists between the ICP power, oxygen flow rate, and RF power. The silicon etch rate decreased with increasing oxygen flow rate due to oxidation of the silicon. The opposite effect on the etching rate was true with PMMA with respect to increasing oxygen flow rate. The silicon etch rate was lower than the PMMA etch rate with low RF power level due to unequal reactivity between the two materials. This unequal atomic activity is logical given that the molar volume for silicon is higher than the polymer, requiring more active species to produce volatile products. Also, at low RF power, the limiting step for the etch rate is not the reaction but the low speed of transport of the reactive gases. This low transport speed becomes more apparent along the course of the reaction as more silicon is exposed. This increased exposure area reacts with the same small amount of transport gases decreasing 
the silicon etch rate. The RF power was increased to overcome this effect and bring the silicon and resist etch rates closer together. With increased RF power, the active species transport limiting step becomes less of a factor and the silicon reaction rate becomes more apparent with increased transport. This is due to the bonds on the silicon surface being weakened by the increased energy of the RIE ion bombardment.

The RIE protocol that gave the best etching rate results were an RF power of 100 $\mathrm{W}$, an ICP power of $200 \mathrm{~W}$, a pressure of $25 \mathrm{mTorr}$ and an oxygen flow rate of $16 \mathrm{sccm}$. The resulting etching rates of silicon and PMMA were close to $7 \mathrm{~nm} / \mathrm{s}$. The etched silicon profiles were measured by atomic force microscopy (AFM). The etched silicon profile and intended design is shown in Figure IV.5 The surface roughnesses were calculated from the raw AFM data separately on the flat areas, sloped areas of the profile, and the top of the radial test, and were averaged together for a complete picture. The completely exposed surroundings are fairly uniform with roughness along the slopes increasing slightly and decreasing again at the least exposed center. This means that radial resist profiles were able to be successfully transferred onto silicon with our setup here in VINSE.

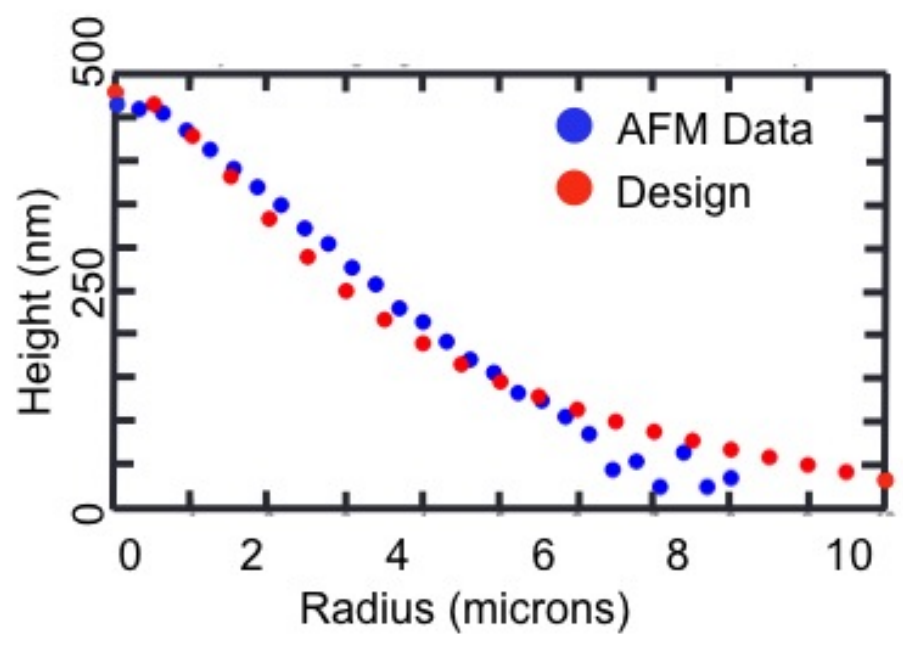

Figure IV.5: Comparison of Designed and FIB Fabricated Maxwell Fisheye Crossing Height Profile

The etched silicon profiles were rougher than the the resist profiles and some edge 
smoothing was observed ${ }^{34}$ as shown in Figure IV.6. This can be attributed to possible imperfect anisotropy from the RIE process. The degree of the slopes can be affected if the lateral etching is not the same as the vertical component, making sharp features like the edges and the intersection between the lens and the background vary from the original design. One more observation is that the structures also tended to shift down into the silica underlayer when overetched. Great care must be taken during fabrication to ensure that these deviations from the designed structures do not potentially affect the waveguide crossing performance.

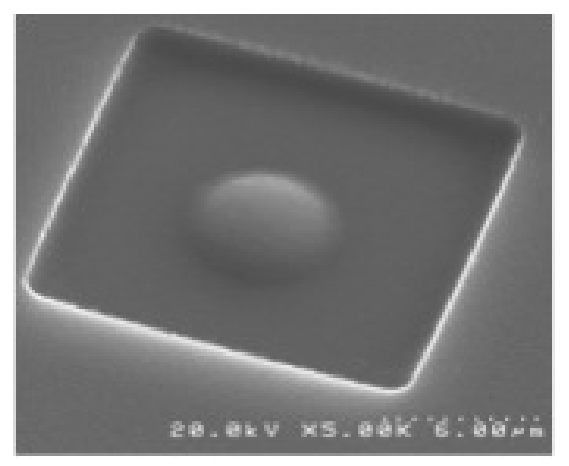

Figure IV.6: Grayscale EBL-RIE of Arbitrary Radially Symmetric Design

In this section, we discussed a novel grayscale fabrication method developed using a computer-generated bitmap pattern with 256 gray levels. This bitmap was then translated into FIB or EBL dose levels, making three-dimensional structures. This method increased the scope of milled materials from binary to arbitrary height profiles, perfect for our application. In this chapter, we discussed the conversion of design height to actual device height via bitmap translation. The resulting bitmaps were used in focused ion beam (FIB) and electron-beam lithography (EBL). Both types of fabrication have their advantages and challenges, which we discussed. Finally, we discussed the agreement of final FIB fabrication to designed MFE waveguide crossing. 


\section{CHAPTER V}

\section{Conclusions and Future Work}

The focusing of the Maxwell fisheye realized by effective waveguide theory has been demonstrated. This new type of waveguide crossing was shown to retain high transmission efficiency in a small crossing area while crossing several waveguides simultaneously in a two-dimensional computer simulation. Two-dimensional mathematical computer simulations were used to predict the behavior of the Maxwell fisheye gradient index lens as a compact, efficient waveguide hub crossing. More work in three-dimensional mathematical computer simulations is needed to inspect the performance of the Maxwell fisheye waveguide crossing. The performance of this waveguide crossing will be confirmed with transmission and crosstalk found experimentally in future work.

In order to obtain experimental results, it is imperative to fabricate both the smooth grayscale Maxwell fisheye waveguide crossing and the sharp steps of the waveguides themselves. For the smooth crossing, two fabrication methods of the Maxwell fisheye with tapered height waveguides with effective waveguide theory were demonstated in this paper. Combining the smooth grayscale patterning with traditional step waveguide patterning proved to be an involved process which will require future work. Fortunately, the ground work for fabrication processes utilizing either grayscale focused ion beam lithography (FIB) or grayscale electron beam lithography (EBL) has been discussed in detail. With future work in simulation, fabrication, and experimental testing, the waveguide crossing can be shown to produce a compact, efficient waveguide hub crossing that is able to be fabricated with straightforward CMOS fabrication techniques. 


\section{Appendix A}

\section{Appendix A: Slab Waveguide Theory}

\section{A.1 Symmetric Waveguides}

Waveguides consist of a high-index dielectric core material surrounded by dielectric mate-

rial or materials of a lower refractive index also known as cladding ${ }^{45,46}$. Light propogates along the length of the waveguide by total internal reflection off of the interfaces between the lower refractive indices of the cladding and core. The higher refractive index dielectric core is used to confine the beam into guided modes, which propogate with no loss. In this section, we will discuss TE and TM modes in asymmetric and symmetric slab waveguides. For more information, please consult Yariv ${ }^{45}$ and Pollock ${ }^{46}$. These modes can be characterized by solving Maxwell's equations for light traveling along the waveguide based on the geometry of the particlar waveguide system which are characterized by the following equations:

$$
\begin{gathered}
\nabla \times H=i \omega \varepsilon_{o} n^{2} E \\
\nabla \times E=-i \omega \mu H
\end{gathered}
$$

In a symmetric waveguide system, the refractive index both above and below the high index core is the same as shown in Figure A.1 below. In Figure A.1, $n_{f}$ is the refractive index of the high-index core waveguide material and $n_{s}$ is the refractive index of the lowerindex surrounding material. In this figure, $h$ is the height of the waveguide along the $x$ direction. The mode of this waveguide would propogate along the $z$ direction.

For the symmetric waveguide case, the plane wave equations are the following:

$$
\begin{aligned}
& E(x, t)=E_{m}(x) e^{i(\omega t-\beta z)} \\
& H(x, t)=H_{m}(x) e^{i(\omega t-\beta z)}
\end{aligned}
$$




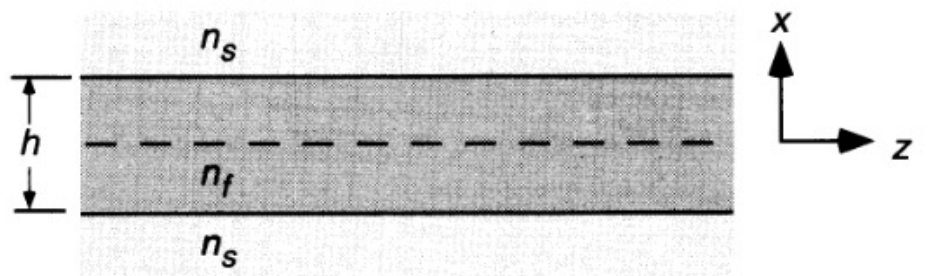

Figure A.1: Symmetric Waveguide Schematic ${ }^{46}$

where $m$ is the mode number, $E$ is the electric field, $H$ is the magnetic field, $x$ is the position along the height of the waveguide core, $z$ is the distance along the propogation length of the material, $\omega$ is the excitation frequency along $t$ time, and $\beta$ is the propogation constant, which is the component of the mode along the length of the waveguide. Two boundary conditions are necessary in order to completely solve these equations. First, the tangental field components at each interface must be equal for continuity. When these components are equal at the interface, the combination of Equations A.4 in terms of the electric field at the boundary, they are represented by:

$$
\left(\frac{\delta^{2}}{\delta x^{2}}+\frac{\delta^{2}}{\delta y^{2}}\right) E(x, y)+\left[k_{o}^{2} n^{2} n(r)-\beta^{2}\right] E(x, y)=0
$$

where $k_{0}$ is the vacuum wavenumber or $\omega / c$ and $n$ is the refractive index as a function of radius.

The second boundary condition requires that the field decays into the surrounding media. This means that the 2 nd term of Equation A.5, or $\left[k_{o}^{2} n^{2} n(r)-\beta^{2}\right]$, is negative outside of the high index waveguide layer and a maximum inside the high index waveguide layer. To describe the decay in the surroundings, $\beta$ becomes:

$$
\begin{gathered}
\beta>k_{o} n \\
\beta>\frac{\omega n_{1}}{c}
\end{gathered}
$$


To describe the maximum of the field in the waveguide, one must take the second derivative of the field to be negative, which yields:

$$
\begin{gathered}
\beta<k_{o} n \\
\beta<\frac{\omega n_{2}}{c}
\end{gathered}
$$

For light propogating along the waveguide with the electric field transverse to this direction, the vertical refractive index profile is as follows:

$$
n(x)=\left\{\begin{array}{l}
n_{2},|x|<\frac{d}{2} \\
n_{1}, \text { otherwise }
\end{array}\right.
$$

For transverse electric (TE) modes, the magnitude of the electric field is transverse to the propapation along the waveguide with a height of $d$. Only $E y, H z$, and $H x$ exist. The field above the core, inside the core itself, and below the core is as follows

$$
E_{m}(x)=\left\{\begin{array}{l}
A \sin (h x)+B \cos (h x),|x|<\frac{d}{2}, \text { Inside the Core } \\
C e^{-q x}, x>\frac{d}{2}, \text { Above the Core } \\
D e^{q x}, x<\frac{d}{2}, \text { Below the Core }
\end{array} H_{z}=\frac{i}{\omega \mu} \frac{\delta E_{y}}{\delta x}\right.
$$

where $A, B, C$, and $D$ are amplitude coefficients. The constants h and $\mathrm{q}$ are the transverse components in the core layer and the cladding, respectively. They are calulated by

$$
\begin{array}{r}
h=\sqrt{\left[\left(\frac{n_{2} \omega}{c}\right)^{2}-\beta^{2}\right.} \\
q=\sqrt{\left[\left(\beta^{2}-\frac{n_{2} \omega}{c}\right)^{2}\right]}
\end{array}
$$


In order to solve for the electric field $E_{x}$ and magnetic field $H_{x}$ at the top interface ( $x=$ $1 / 2 \mathrm{~d})$ and the bottom interface $(x=-1 / 2 \mathrm{~d})$ of the waveguide, their magnitudes have to be equal at all interfaces. These magnitudes are represented by the following equations:

$$
\begin{array}{r}
E_{x} @ 1 / 2 d: A \sin (h d / 2)+B \cos (h d / 2)=C e^{-q d / 2} \\
E_{x} @-1 / 2 d:-A \sin (h d / 2)+B \cos (h d / 2)=D e^{-q d / 2} \\
H_{z} @ 1 / 2 d: h A \cos (h d / 2)-h B \sin (-h d / 2)=-q C e^{-q d / 2} \\
H_{z} @-1 / 2 d: h A \cos (h d / 2)+h B \sin (-h d / 2)=q D e^{-q d / 2} .
\end{array}
$$

Equations A.17 simplify to the following:

$$
\begin{array}{r}
12 A \sin (h d / 2)=(C-D) e^{-q d / 2} \\
2 B \cos (h d / 2)=(C+D) e^{-q d / 2} \\
2 h A \cos (h d / 2)=-q(C-D) e^{-q d / 2} \\
2 h B \sin (h d / 2)=q(C+D) e^{-q d / 2}
\end{array}
$$

Both the symmetric and antisymmetric modes can be solved by the equation

$$
\tan (h d)=\frac{2 h q}{h^{2}-q^{2}}
$$

The normalized propogation constant of the waveguide, also known as the modal effective refractive index, is described by the following equation:

$$
n_{e f f}=\bar{\beta}=\frac{\beta}{\omega c}=\frac{c}{v_{p}}
$$

where $v_{p}$ is the phase velocity of the mode through the waveguide.

TM modes occur when the magnetic field of the mode propogating through the waveg- 
uide is perpendicular to the plan of propagation. In that case, the fields are characterized as:

$$
\begin{array}{r}
1 H_{y}(x, z, t)=H_{m}(x) e^{i(\omega t-\beta z)} \\
E_{z}=-\frac{i}{\omega \mu} \frac{\delta H_{y}}{\delta x} \\
E_{x}=\frac{i}{\omega \mu} \frac{\delta H_{y}}{\delta z}
\end{array}
$$

where $H_{m}$ is defined as:

$$
H_{m}(x)=\left\{\begin{array}{l}
A \sin (h x)+B \cos (h x),|x|<\frac{d}{2} \\
C e^{-q x}, x>\frac{d}{2} \\
D e^{q x}, x<\frac{d}{2}
\end{array}\right.
$$

Similarly, both symmetric and antisymmetric modes in this case can be characterized with the following equation:

$$
\tan (h d)=\frac{2 h \bar{q}}{h^{2}-\bar{q}^{2}}
$$

where $\bar{q}$ is:

$$
\bar{q}=\frac{n_{2}^{2}}{n_{1}^{2}} q
$$

\section{A.2 Asymmetric Waveguides}

For asymmetric waveguides, the refractive indices above and below the high index core are the not the same. In this case the refractive index profile is as follows:

$$
n(x)=\left\{\begin{array}{l}
n_{1}, 0<x \\
n_{2},-t<x<0 \\
n_{1}, x<-t
\end{array}\right.
$$


where $n_{2}>n_{1}, n_{3}$, and $t$ is the thickness of the waveguide. For TE modes confined within the waveguide core where the electric field is perpendicular to the direction of propagation, the electric field is defined as:

$$
E_{y}(x, y, z, t)=E_{m}(x) e^{i(\omega t-\beta z)}
$$

where $E_{m}$ is:

$$
E_{m}(x)=\left\{\begin{array}{l}
C e^{-q x}, \quad 0 \leq x \\
C\left(\cos (h x)-\frac{q}{h} \sin (h x)\right), \quad-t \leq x \leq 0 \\
C\left(\cos (h t)+\frac{q}{h} \sin (h t)\right) e^{p(x+t)}, x \leq-t .
\end{array}\right.
$$

In this case, we have three transverse constants $h, q$, and $p$. In the symmetric waveguide case, only two constants were needed to describe the transverse field behavior in the core waveguide and on the interfaces of the core waveguide which were identical. This is no longer true for the asymmetric waveguide case. In the asymmetric waveguide case, three transverse components are required to adequately describe the system: $h$ to describe the transverse field inside of the high-index core material with a refractive index of $n_{2}, q$ to describe the transverse field in the material above the core $\left(n_{3}\right)$, and $p$ to describe the transverse field in the material below the core. These three components are calculated by:

$$
\begin{aligned}
& 1 h=\sqrt{\left[\left(\frac{n_{2} \omega}{c}\right)^{2}-\beta^{2}\right.} \\
& q=\sqrt{\left[\left(\beta^{2}-\frac{n_{1} \omega}{c}\right)^{2}\right]} \\
& p=\sqrt{\left[\left(\beta^{2}-\frac{n_{3} \omega}{c}\right)^{2}\right]}
\end{aligned}
$$


Both symmetric and antisymmetric modes in the asymmetric waveguide system can be solved by the equation:

$$
\tan (h t)=\frac{q+p}{h\left(1-\frac{p q}{h^{2}}\right)}
$$

The normalized propogation constant of the waveguide, also known as the modal effective refractive index is can by the following equation

$$
n_{e f f}=\bar{\beta}=\frac{\beta}{\omega c}=\frac{c}{v_{p}}
$$

where $\beta$ is numerically solved for in this case and $v_{p}$ is the phase velocity of the mode through the waveguide.

Transverse magnetic (TM) modes occur when the magnetic field of the mode propogating through the waveguide is perpendicular to the plane of propagation. In that case, the fields are characterized as:

$$
\begin{array}{r}
1 H_{y}(x, z, t)=H_{m}(x) e^{i(\omega t-\beta z)} \\
E_{x}=\frac{i}{\omega \mu} \frac{\delta H_{y}}{\delta z}=\frac{\beta}{\omega \varepsilon} H_{m}(x) e^{i(\omega t-\beta z)} \\
E_{z}=-\frac{i}{\omega \mu} \frac{\delta H_{y}}{\delta x}
\end{array}
$$

where $H_{m}$ is defined as: $H_{m}(x)=$

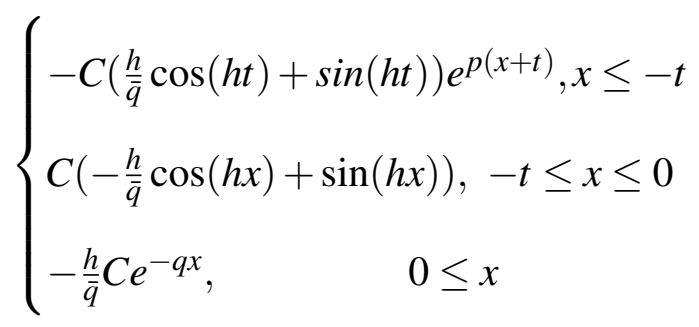

where $C, q$, and $p$ are the same as previous examples. Two new variables $\bar{p}$ and $\bar{q}$ describe the transverse magnetic field component in the top cladding and the bottom cladding, re- 
specitively. They are described by the following equations:

$$
\begin{aligned}
& \bar{p}=\frac{n_{2}^{2}}{n_{3}^{2}} p \\
& \bar{q}=\frac{n_{2}^{2}}{n_{1}^{2}} q .
\end{aligned}
$$

Both symmetric and asymmetric modes for the asymmetric waveguide system in transverse magnetic operation can be defined by:

$$
\tan (h t)=\frac{h(\bar{p}+\bar{q})}{\left(h^{2}-\bar{p} \bar{q}\right)}
$$

An important difference to note between the symmetric and asymmetric TE/TM cases is the relationship between waveguide thicknesses and modal propogation. For asymmetric waveguides, there is a particular thickness to wavelength ratio above which modes can propogate. This is known as the cutoff thickness. For symmetric waveguides, there is no cutoff waveguide thickness to wavelength ratio after which modes can propogate. These thickness for $m^{\text {th }}$ mode can be calculated by:

$$
\begin{aligned}
& \left(\frac{t}{\lambda}\right)_{T E}=\frac{1}{2 \pi \sqrt{n_{2}^{2}-n_{3}^{2}}}\left[m \pi+\sqrt{\tan ^{-1}\left(\frac{n_{3}^{2}-n_{1}^{2}}{n_{2}^{2}-n_{3}^{2}}\right)}\right] \\
& \left(\frac{t}{\lambda}\right)_{T M}=\frac{1}{2 \pi \sqrt{n_{2}^{2}-n_{1}^{2}}}\left[m \pi+\sqrt{\tan ^{-1}\left(\frac{n_{3}^{2}-n_{1}^{2}}{n_{2}^{2}-n_{3}^{2}}\right)}\right]
\end{aligned}
$$




\section{REFERENCES}

[1]G Moore. Cramming more components onto integrated circuits. Electronics, 38(8), 1965.

[2]Scott E. Thompson and Srivatsan Parthasarathy. Moore's law: the future of si microelectronics. Materials Today, 9(6):20 - 25, 2006.

[3]A. Oishi, O. Fujii, T. Yokoyama, K. Ota, T. Sanuki, H. Inokuma, K. Eda, T. Idaka, H. Miyajima, S. Iwasa, H. Yamasaki, K. Oouchi, K. Matsuo, H. Nagano, T. Komoda, Y. Okayama, T. Matsumoto, K. Fukasaku, T. Shimizu, K. Miyano, T. Suzuki, K. Yahashi, A. Horiuchi, Y. Takegawa, K. Saki, S. Mori, K. Ohno, I. Mizushima, M. Saito, M. Iwai, S. Yamada, N. Nagashima, and F. Matsuoka. High performance cmosfet technology for $45 \mathrm{~nm}$ generation and scalability of stress-induced mobility enhancement technique. pages 229-232, 2005.

[4]H. Ohta, Y. Kim, Y. Shimamune, T. Sakuma, A. Hatada, A. Katakami, T. Soeda, K. Kawamura, H. Kokura, H. Morioka, T. Watanabe, J.O.Y. Hayami, J. Ogura, M. Tajima, T. Mori, N. Tamura, M. Kojima, and K. Hashimoto. High performance $30 \mathrm{~nm}$ gate bulk cmos for $45 \mathrm{~nm}$ node with /spl sigma/-shaped sige-sd. pages 4 pp.-240, 2005.

[5]N.S. Kim, T. Austin, D. Baauw, T. Mudge, K. Flautner, J.S. Hu, M.J. Irwin, M. Kandemir, and V. Narayanan. Leakage current: Moore's law meets static power. Computer, 36(12):68-75, 2003.

[6]Qian Li-Bo, Zhu Zhang-Ming, and Yang Yin-Tang. Through-silicon-via-aware interconnect prediction model for 3D integrated circuirt. Acta Physica Sinica, 61(6), Mar 2012.

[7]KY Chen, WD Brown, LW Schaper, SS Ang, and HA Naseem. A study of the high frequency performance of thin film capacitors for electronic packaging. IEEE Transactions On Advanced Packaging, 23(2):293-302, MAY 2000.

[8]P Larsson. Parasitic resistance in an MOS transistor used as on-chip decoupling capacitance. IEEE Journal Of Solid-State Circuits, 32(4):574-576, Apr 1997.

[9]Ayc Yu. Electron Tunneling and Contact Resistance of Metal-Silicon Contact Barriers. Solid-State Electronics, 13(2):239-\&, 1970.

[10]W. Bogaerts, D. Taillaert, B. Luyssaert, P. Dumon, J. Van Campenhout, P. Bienstman, D. Van Thourhout, R. Baets, V. Wiaux, and S. Beckx. Basic structures for photonic integrated circuits in silicon-on-insulator. Opt. Express, 12(8):1583-1591, Apr 2004.

[11]RA SOREF. Silicon-Based Optoelectronics. Proceedings of The IEEE, 81(12):1687-1706, Dec 1993.

[12]Richard Soref. The past, present, and future of silicon photonics. IEEE Journal of Selected Topics In Quantum Electronics, 12(6, 2):1678-1687, Nov-Dec 2006.

[13]Bahrain Jalali and Sasan Fathpour. Silicon photonics. Journal of Lightwave Technology, 24(12):4600-4615, Dec 2006. 
[14]C Manolatou, SG Johnson, SH Fan, PR Villeneuve, HA Haus, and JD Joannopoulos. Highdensity integrated optics. Journal of Lightwave Technology, 17(9):1682-1692, SEP 1999.

[15]Y Taur, DA Buchanan, W Chen, DJ Frank, KE Ismail, SH Lo, GA SaiHalasz, RG Viswanathan, HJC Wann, SJ Wind, and HS Wong. CMOS scaling into the nanometer regime. Proceedings of The IEEE, 85(4):486-504, Apr 1997.

[16]Fengnian Xia, Lidija Sekaric, and Yurii Vlasov. Ultracompact optical buffers on a silicon chip. Nature Photonics, 1(1):65-71, Jan 2007.

[17]Gunther Roelkens, Liu Liu, Di Liang, Richard Jones, Alexander Fang, Brian Koch, and John Bowers. III-V/silicon photonics for on-chip and inter-chip optical interconnects. Laser \& Photonics Reviews, 4(6):751-779, Nov 2010.

[18]Thijs Spuesens, Fabien Mandorlo, Pedro Rojo-Romeo, Philippe Regreny, Nicolas Olivier, Jean-Marc Fedeli, and Dries Van Thourhout. Compact Integration of Optical Sources and Detectors on SOI for Optical Interconnects Fabricated in a $200 \mathrm{~mm}$ CMOS Pilot Line. Journal Of Lightwave Technology, 30(11):1764-1770, JUN 12012.

[19]Martijn J. R. Heck, Hui-Wen Chen, Alexander W. Fang, Brian R. Koch, Di Liang, Hyundai Park, Matthew N. Sysak, and John E. Bowers. Hybrid Silicon Photonics for Optical Interconnects. IEEE Journal Of Selected Topics In Quantum Electronics, 17(2):333346, Mar-Apr 2011.

[20]Di Liang, Alexander W. Fang, Hui-Wen Chen, Matthew N. Sysak, Brian R. Koch, Erica Lively, Omri Raday, Ying-Hao Kuo, Richard Jones, and John E. Bowers. Hybrid silicon evanescent approach to optical interconnects. Applied Physics A-Materials Science \& Processing, 95(4):1045-1057, Jun 2009.

[21]H Takahashi, K Oda, and H Toba. Impact of crosstalk in an arrayed-waveguide multiplexer on NxN optical interconnection. Journal of Lightwave Technology, 14(6):1097-1105, Jun 1996.

[22]YP Li and $\mathrm{CH}$ Henry. Silica-based optical integrated circuits. IEE ProceedingsOptoelectronics, 143(5):263-280, OCT 1996.

[23]SG Johnson, C Manolatou, SH Fan, PR Villeneuve, JD Joannopoulos, and HA Haus. Elimination of cross talk in waveguide intersections. Optics Letters, 23(23):1855-1857, Dec 11998.

[24]Pablo Sanchis, Pablo Villalba, Francisco Cuesta, Andreas Hakansson, Amadeu Griol, Jose V. Galan, Antoine Brimont, and Javier Marti. Highly efficient crossing structure for silicon-on-insulator waveguides. Optics Letters, 34(18):2760-2762, SEP 152009.

[25]Junbo Feng, Qunqing Li, and Shoushan Fan. Compact and low cross-talk silicon-oninsulator crossing using a periodic dielectric waveguide. Opt. Lett., 35(23):3904-3906, Dec 2010. 
[26]Andrei V. Tsarev. Efficient silicon wire waveguide crossing with negligible loss and crosstalk. Opt. Express, 19(15):13732-13737, Jul 2011.

[27]Wim Bogaerts, Pieter Dumon, Dries Van Thourhout, and Roel Baets. Low-loss, lowcross-talk crossings for silicon-on-insulator nanophotonic waveguides. Opt. Lett., 32(19):2801-2803, Oct 2007.

[28]Weiqiang Ding, Donghua Tang, Yan Liu, Lixue Chen, and Xiudong Sun. Compact and low crosstalk waveguide crossing using impedance matched metamaterial. Applied Physics Letters, 96(11):111114, 2010.

[29]Y Jiao, SF Mingaleev, M Schillinger, DAB Miller, S Fan, and K Busch. Wannier basis design and optimization of a photonic crystal waveguide crossing. IEEE Photonics Technology Letters, 17(9):1875-1877, SEP 2005.

[30]Aleksandr Biberman, Kyle Preston, Gilbert Hendry, Nicolas Sherwood-Droz, Johnnie Chan, Jacob S. Levy, Michal Lipson, and Keren Bergman. Photonic Network-on-Chip Architectures Using Multilayer Deposited Silicon Materials for High-Performance Chip Multiprocessors. ACM Journal On Emerging Technologies In Computing Systems, 7(2, SI), JUN 2011.

[31]Alberto Scandurra. Silicon Photonics: The System on Chip Perspective. In Lockwood, DJ and Pavesi, L, editor, Silicon Photonics Ii: Components And Integration, volume 119 of Topics in Applied Physics, pages 143-168. Springer-Verlag Berlin, Heidelberger Platz 3, D-14197 Berlin, Germany, 2011.

[32]Xia Chen, Chao Li, and Hon K. Tsang. Device engineering for silicon photonics. NPG Asia Materials, 3(1):34-40, JAN 2011.

[33]S. Azimi, J. Song, Z. Y. Dang, H. D. Liang, and M. B. H. Breese. Three-dimensional silicon micromachining. Journal of Micromechanics And Microengineering, 22(11), NOV 2012.

[34]Joy C. Garnett and Jason G. Valentine. Maxwell fisheye lens as a waveguide crossing for integrated photonics. In Conference on Lasers and Electro-Optics 2012, page JW4A.88. Optical Society of America, 2012.

[35]C. A. (alias J.C. Maxwell) Swainson. Maxwell fisheye lens as a waveguide crossing for integrated photonics. Camb. Dublin. Math. J, 8:188-189, 1854.

[36]Ulf Leonhardt. Perfect imaging without negative refraction. New Journal of Physics, 11(9):093040, 2009.

[37]Juan C Gonzlez, Pablo Bentez, and Juan C Miano. Perfect drain for the maxwell fish eye lens. New Journal of Physics, 13(2):023038, 2011.

[38]Paul Kinsler, Jiajun Tan, Timothy C. Y. Thio, Claire Trant, and Navin Kandapper. Maxwell's fishpond. European Journal of Physics, 33(6):1737-1750, Nov 2012. 
[39]Ulf Leonhardt and Thomas G. Philbin. Chapter 2 transformation optics and the geometry of light. In E Wolf, editor, Progress in Optics, volume 53 of Progress in Optics, pages 69 - 152. Elsevier, 2009.

[40]S. Guenneau, A. Diatta, and R.C. McPhedran. Focusing: coming to the point in metamaterials. Journal of Modern Optics, 57(7):511-527, 2010.

[41]Yongqi $\mathrm{Fu}$ and Bryan Kok Ann Ngoi. Virtual gray-scale mask for fabrication of micro-optical elements via focused ion beam direct writing. Optical Engineering, 44(12):128002-128002-5, 2005.

[42]M. D. Henry, M. J. Shearn, B. Chhim, and A. Scherer. Ga+ beam lithography for nanoscale silicon reactive ion etching. Nanotechnology, 21(24), Jun 182010.

[43]Arne Schleunitz and Helmut Schift. Fabrication of 3d patterns with vertical and sloped sidewalls by grayscale electron-beam lithography and thermal annealing. Microelectronic Engineering, 88(8):2736 - 2739, 2011. ¡ce:title ¿Proceedings of the 36th International Conference on Micro- and Nano-Engineering (MNE);/ce:title $i ; x_{\text {xocs:full-name }} 36$ th International Conference on Micro- and Nano-Engineering (MNE);/xocs:full-name $\underset{i}{ }$.

[44]Guido Piaszenski, Ulrich Barth, Axel Rudzinski, Andreas Rampe, Andreas Fuchs, Markus Bender, and Ulrich Plachetka. 3D structures for UV-NIL template fabrication with grayscale e-beam lithography. Microelectronic Engineering, 84(5-8):945-948, MayAug 2007. 32nd International Conference on Micro- and Nano-Engineering, Barcelona, SPAIN, SEP 17-20, 2006.

[45]Amnon Yariv and Pochi Yeh. Photonics: Optical Electronics in Modern Communications (The Oxford Series in Electrical and Computer Engineering). Oxford University Press, Inc., 2006.

[46]Clifford R Pollock. Fundamentals of optoelectronics. Irwin Chicago, 1995. 